\title{
GENERALIZED HERMITE INTERPOLATION VIA MATRIX-VALUED CONDITIONALLY POSITIVE DEFINITE FUNCTIONS
}

\author{
FRANCIS J. NARCOWICH AND JOSEPH D. WARD
}

\begin{abstract}
In this paper, we consider a broad class of interpolation problems, for both scalar- and vector-valued multivariate functions subject to linear side conditions, such as being divergence-free, where the data are generated via integration against compactly supported distributions. We show that, by using certain families of matrix-valued conditionally positive definite functions, such interpolation problems are well poised; that is, the interpolation matrices are invertible. As a sample result, we show that a divergence-free vector field can be interpolated by a linear combination of convolutions of the data-generating distributions with a divergence-free, $3 \times 3$ matrix-valued conditionally positive definite function. In addition, we obtain norm estimates for inverses of interpolation matrices that arise in a class of multivariate Hermite interpolation problems.
\end{abstract}

\section{INTRODUCTION}

Background. The last few years have seen an increase in our theoretical understanding of methods for dealing with the problem of interpolating function values when the data sites are both scattered and multidimensional. Duchon's work on the method of thin-plate splines [5, 6] and the work of Micchelli [18] and Madych and Nelson [15, 16] on radial basis functions (RBFs) established the invertibility of the interpolation matrices associated with these functions; that is, their work established that the scattered-data interpolation problem was well poised-i.e., a solution to the problem exists and is unique-relative to the families of either the thin-plate splines or certain RBFs, such as the Hardy multiquadrics $[10,11]$ or the Gaussians. The Hardy multiquadrics have been applied extensively in surface fitting problems arising in geodesy, geophysics, and other areas [11], while the Gaussians have been employed in problems arising in connection with neural networks [1, 21,22] and adaptive control [24].

A method for dealing with a Hermite interpolation problem - the problem of interpolation of data involving both function values and values of combinations of derivatives-when the data come from scattered, multidimensional sites was introduced by Hardy [11], who mentioned that he had had computational success with it. This method also arises at least implicitly in connection with the

Received by the editor October 27, 1992 and, in revised form, September 14, 1993.

1991 Mathematics Subject Classification. Primary 41A05, 41A63, 41A29.

Key words and phrases. Conditionally positive definite, RBF, divergence-free interpolant.

Supported by the Air Force Office of Scientific Research under Grant Number F49620-92-J0403DEF. 
collocation methods for numerically solving PDEs introduced by Kansa [13, 14].

In [12], Jetter, Riemenschneider, and Shen considered the problem of cardinal Hermite interpolation on the lattice $\mathbb{Z}^{d}$ by a suitable basis of functions. They employed a matrix-valued symbol, whose determinant they proved was nonvanishing - provided only mild restrictions were met-, and thereby established that the Hermite interpolation problems under consideration were well poised. The basis utilized was generated by multiplying functions with suitable decay by certain trigonometric polynomials. There are many RBFs, Gaussians for example, that fall into the class of functions having the appropriate decay properties; however, the popular Hardy multiquadrics apparently do not. The arguments in [12] involve the lattice in an inherent way, and hence they do not apply to the scattered-data setting.

$\mathrm{Wu}$ [26], who used Kriging methods and a basis generated by a function $\varphi: \mathbb{R}^{s} \rightarrow \mathbb{R}$ having a distributional Fourier transform that agreed with a positive function $\hat{\varphi}$ on $\mathbb{R}^{s} \backslash\{0\}-$ Gaussians and Hardy multiquadrics are such $\varphi$ discussed a broad class of scattered-data Hermite-type interpolation problems in terms of their being well poised. Specifically, Wu dealt with the following situation: Let $\left\{x_{1}, \ldots, x_{m}\right\}$-the set of centers-be a finite subset of $\mathbb{R}^{s}$, and let $\left\{L_{1}, \ldots, L_{m}\right\}$ be a set of linearly independent linear functionals involving linear combinations of point evaluations, differentiations, and difference operators that are evaluated on some of the centers. The problem is to interpolate data generated by the $L_{j}$ 's with the additional requirement that the method employed reproduces exactly any degree- $(k-1)$ polynomial that generates the data. To simplify matters, we will describe only the problem without any requirement concerning polynomial reproduction. Consider functions of the form $s(x):=\sum_{j=1}^{m} \lambda_{j} L_{j}^{y}[\varphi(x-y)]$, where $L_{j}^{y}$ acts on the variable $y$, and the $\lambda_{j}$ 's are arbitrary complex numbers. The main result in [26] is that if $d_{1}, \ldots, d_{m}$ are arbitrary complex numbers, and if certain integrals that involve $\hat{\varphi}$ exist, then there exist unique constants $\lambda_{1}, \ldots, \lambda_{m}$ such that $s$ satisfies $L_{j}(s)=d_{j}$ for $j=1, \ldots, m$. Thus, for example, if $s=1, m=2$, and $f$ is a smooth function, then the linear functionals $L_{1}(f)=f\left(x_{2}\right)+f^{\prime}\left(x_{1}\right)$ and $L_{2}(f)=f\left(x_{1}\right)$ can be used to form the set $\left\{L_{1}, L_{2}\right\}$. It should, however, be noted that the number of linear functionals must equal the number of centers. Thus, to use the results in [26] to solve the problem in which $L_{1} f=f\left(x_{1}\right)$ and $L_{2}(f)=f^{\prime}\left(x_{1}\right)$, one must introduce a second center $x_{2} \neq x_{1}$.

For a lucid, concise discussion of the results in [12] and [26], see Buhmann [4].

An example. In order to clarify the generalized Hermite interpolation problems that we will deal with in this paper, we will first look at an illustrative example involving fluid flow; terms are from R. Meyer's book [17].

An incompressible fluid that has reached its steady state is described by a smooth, time-independent vector field on $\mathbb{R}^{3}$, the fluid's velocity, $\mathbf{v}(\mathbf{x})=$ $(p(\mathbf{x}), q(\mathbf{x}), r(\mathbf{x}))$, where $\mathbf{x}:=(x, y, z)$. Because the flow is incompressible, $\mathbf{v}$ is divergence-free; that is, $\nabla \cdot \mathbf{v}(\mathbf{x}) \equiv 0$.

Consider the following interpolation problem for an incompressible fluid. The data available consists of the following:

(i) $d_{1}=p\left(\mathbf{x}_{1}\right)$, the $x$-component of $\mathbf{v}$ at a point $\mathbf{x}_{1}$ in $\mathbb{R}^{3}$; 
(ii) $d_{2}=r\left(\mathbf{x}_{2}\right)$, the $z$-component of $\mathbf{v}$ at $\mathbf{x}_{2} \neq \mathbf{x}_{1}$;

(iii) $d_{3}=\nabla \times \mathbf{v}\left(\mathbf{x}_{1}\right) \cdot \mathbf{k}=q_{x}\left(\mathbf{x}_{1}\right)-p_{y}\left(\mathbf{x}_{1}\right)$, the $z$-component of the vorticity;

(iv) $d_{4}=\iint_{S} \mathbf{v} \cdot \mathbf{n} d S$, the flux over $S$, the hemisphere $x^{2}+y^{2}+z^{2}=1$, $z \geq 0$, with orientation chosen so that $\mathbf{n} \cdot \mathbf{k} \geq 0$.

Regard the vectors involved as columns. It is convenient to rewrite the data in terms of these compactly supported column-vector distributions:

$$
\begin{gathered}
\phi_{1}(\mathbf{x}):=\left(\begin{array}{c}
\delta\left(\mathbf{x}-\mathbf{x}_{1}\right) \\
0 \\
0
\end{array}\right), \quad \phi_{2}(\mathbf{x}):=\left(\begin{array}{c}
0 \\
0 \\
\delta\left(\mathbf{x}-\mathbf{x}_{2}\right)
\end{array}\right), \\
\phi_{3}(\mathbf{x}):=\left(\begin{array}{c}
\frac{\partial}{\partial y} \delta\left(\mathbf{x}-\mathbf{x}_{1}\right) \\
-\frac{\partial}{\partial x} \delta\left(\mathbf{x}-\mathbf{x}_{1}\right) \\
0
\end{array}\right), \quad \phi_{4}(\mathbf{x}):=\left(\begin{array}{c}
\frac{x \chi(x, y)}{\sqrt{1-x^{2}-y^{2}}} \delta\left(z-\sqrt{1-x^{2}-y^{2}}\right) \\
\frac{y \chi(x, y)}{\sqrt{1-x^{2}-y^{2}}} \delta\left(z-\sqrt{1-x^{2}-y^{2}}\right) \\
\chi(x, y) \delta\left(z-\sqrt{1-x^{2}-y^{2}}\right)
\end{array}\right) .
\end{gathered}
$$

Here, $\chi(x, y)$ is the characteristic function for $\left\{(x, y) \in \mathbb{R}^{2}: x^{2}+y^{2} \leq 1\right\}$, and $\delta$ is the Dirac delta function. In terms of these distributions, the data set becomes

$$
d_{j}=\int_{\mathbb{R}^{3}} \phi_{j}^{T}(\mathbf{x}) \mathbf{v}(\mathbf{x}) d^{3} x, \quad j=1, \ldots, 4 .
$$

Let $\mathfrak{G}_{\text {div }}$ denote the set of all $C^{\infty}$ vector fields that are divergence-free. It is important to note when the compactly supported distributions generating this data act on vector fields in $\mathfrak{G}_{\text {div }}$, they are linearly independent. If $S$ were closed, the divergence theorem would imply that $\phi_{4}$ would vanish on $\mathfrak{G}_{\text {div }}$; the $\phi_{j}$ 's would not be linearly independent in this case. We will say that a set of compactly supported distributions is $\mathfrak{G}_{\text {div }}$-linearly independent if the set is linearly independent when restricted to functions in $\mathfrak{G}_{\text {div }}$.

Using a vector field from $\mathfrak{G}_{\text {div }}$, so that the incompressible character of the fluid is preserved, we wish to interpolate the data given above. To do this, we will use a matrix-valued "RBF" that we construct in $\S 6$ :

$$
h_{t}^{3 \times 3}(\mathbf{x})=\left\{\left(4 t-4 t^{2}\|\mathbf{x}\|^{2}\right) I+4 t^{2} \mathbf{x x}^{T}\right\} e^{-t\|\mathbf{x}\|^{2}},
$$

where $t>0$ is a parameter at our disposal. It is easily shown that the columns of $h_{t}^{3 \times 3}$ are divergence-free vector fields. The candidates for interpolants will be taken to have the form

$$
\mathbf{V}(\mathbf{x})=\sum_{j=1}^{4} c_{j} \int_{\mathbb{R}^{3}} h_{t}^{3 \times 3}\left(\mathbf{x}-\mathbf{x}^{\prime}\right) \phi_{j}\left(\mathbf{x}^{\prime}\right) d^{3} \mathbf{x}^{\prime}, \quad \text { where } c_{j} \in \mathbb{R}, j=1, \ldots, 4 .
$$

Apart from the implied matrix multiplication, the integral above is the usual convolution product for distributions $[8,25]$. If we denote convolution by $*$ and we are careful about the order in which quantities are multiplied, we may write the last equation as

$$
\mathbf{V}(\mathbf{x})=\sum_{j=1}^{4} c_{j} h_{t}^{3 \times 3} * \phi_{j}(\mathbf{x})
$$

One may show by direct computation that $\mathbf{V}(\mathbf{x})$ is a vector field in $\mathfrak{G}_{\text {div }}$. 
Can we choose the coefficients $c_{j}$ so that the vector-valued function $\mathbf{V}$ interpolates the data, for an arbitrary choice of the $d_{j}$ 's? This question is equivalent to the question of whether the $4 \times 4$ matrix $A$, with entries

$$
A_{k, j}:=\int_{\mathbb{R}^{3}} \phi_{k}^{T}(\mathbf{x}) h_{t}^{3 \times 3} * \phi_{j}(\mathbf{x}) d^{3} x,
$$

is invertible. In fact, it is a consequence of our method of constructing $h_{t}^{3 \times 3}$ that $A$ is positive definite, and therefore invertible. Thus, if the coefficient vector is $c=A^{-1} d$, then $\mathbf{V}$ will interpolate the data.

The matrix-valued nature of $h_{t}^{3 \times 3}$ is essential if we wish our interpolants to be divergence-free. One can show, using Fourier transform theory, that it is impossible to produce such a vector field with scalar RBFs. A second feature is that the compact distributions employed were linearly independent when restricted to the space $\mathfrak{G}_{\text {div }}$. If this were not the case, $A$ would have been singular. It should be stressed here that one may have linear independence relative to all $C^{\infty}$ vector fields, but not relative to the divergence-free vector fields. As we mentioned above, if the surface $S$ used to define $\phi_{4}$ were closed, then for all $\mathbf{v} \in \mathfrak{G}_{\text {div }}$ we would have $\int_{\mathbb{R}^{3}} \phi_{4}^{T}(x) \mathbf{v}(x) d^{3} x=0$. On the other hand, as long as the compact distributions used are linearly independent when restricted to $\mathfrak{G}_{\text {div }}$, they can be arbitrary; there is no restriction to point evaluationswhence our ability to interpolate fluxes. The space $\mathfrak{G}_{\text {div }}$ has a very important feature that allows us to work in it: $\mathfrak{G}_{\text {div }}$ has the property that if $\mathbf{v} \in \mathfrak{G}_{\text {div }}$, then $\mathbf{v} * \varphi$ is also in $\mathfrak{G}_{\text {div }}$ for any scalar-valued compact distribution $\varphi$. Finally, this example provides us with a good illustration of what a "generalized Hermite interpolation problem" is, what it is good for, and what it means for such a problem to be well poised.

Outline and summary. Our purpose in writing this paper is three-fold. First, we want to provide a distributional framework within which one may study questions of whether generalized Hermite interpolation problems are well poised and whether they are stable. The problems that we address have data generated by compactly supported distributions integrated against a $C^{\infty}$ function $f: \mathbb{R}^{s} \rightarrow \mathbb{C}^{n}$, where $f$ may satisfy side conditions (e.g., $\nabla \cdot f \equiv 0$ ) that amount to the $f$ 's belonging to an admissible subspace $\mathfrak{G}$ (see Definition 1.1) of the $C^{\infty}$ functions. Such data of course can be generated by distributions that are point evaluations, difference operators, or differential operators applied to a scalar-valued $f$, and so the problems treated in [26] are included among those that we deal with here. The interpolant solving such a problem is not only required to reproduce the data, but it is also required to be in $\mathfrak{G}$ and to agree with a polynomial of some predetermined degree when that polynomial generates the data. To set up this framework, we introduce the concept of a matrix-valued, $\mathfrak{G}$-conditionally positive definite function ( $\mathfrak{G}-\mathrm{CPDF}$ ) of order $m$; this concept generalizes the scalar-valued version introduced by Gelfand and Vilenkin [9] and refined by Madych and Nelson [16]. In $\S 1$, we establish notation, discuss admissible subspaces, and define matrix-valued order- $m \mathfrak{G}$ CPDFs. At the beginning of $\S 2$, we describe in detail the generalized Hermite interpolation problems that we are dealing with; the interpolants used in these problems are linear combinations of convolutions of the vector-valued, datagenerating distributions with an order- $m$, matrix-valued $\mathfrak{G}-\mathrm{CPDF}$. It is also in 
$\S 2$ that we provide a unifying framework for solving such problems. The linchpin of that framework is Theorem 2.3, which establishes that these problems are well poised under two sets of conditions: mild restrictions on the dimensions of certain spaces and the condition that the order- $m$, matrix-valued $\mathfrak{G}$-CPDF be a strictly $\mathfrak{G}-\mathrm{CPDF}$. (See $\S 1$.)

Second, we wish to use our framework to demonstrate that a broad class of generalized Hermite interpolation problems are well poised. Essentially, this entails establishing the existence of an order- $m$ strictly $\mathfrak{G}$-CPD function for a given $\mathfrak{G}$. The first step in carrying out this task is to obtain explicit forms for order- $m$ matrix-valued CPDFs. (CPDF $=\mathfrak{G}$-CPDF when $\mathfrak{G}$ is the whole space; i.e., there are no side conditions.) This we do in $\S 3$. In $\S 4$, we obtain an explicit characterization of certain distributions that play a very important role in our work. These dstributions are related to admissible subspacces of smooth functions that satisfy homogeneous, constant-coefficient partial differential equations. In $\S 5$, we characterize order- $m$ matrix-valued CPDFs that are also $\mathfrak{G}$-CPDFs, provided $\mathfrak{G}$ is one of the admissible spaces treated in $\S 4$. In $\S 6$, we give sufficient conditions for an order- $m$ G-CPDF to be strictly $\mathfrak{G}$ $\mathrm{CPD}$, again assuming that $\mathfrak{G}$ is as in $\S 4$. Indeed, we give explicit methods for constructing such functions, and thereby both establish that a wide class of generalized Hermite interpolation problems, including those studied in [26], are well poised, and provide a variety of tools for solving Hermite interpolation problems in the presence of side conditions. In particular, in $\S 6$ we construct the "divergence-free RBF" discussed in our example above.

Third, we will use our distribution-theoretic framework to study the stability of certain mutivariate Hermite interpolation problems. In particular, in $\S 7$ we obtain estimates on the norm of the inverse of the scattered-data interpolation matrix that is associated with interpolating values and certain multiples of directional derivatives for a scalar-valued function defined on $\mathbb{R}^{s}$. The main result is that these estimates are governed by the same parameters as in the case of ordinary interpolation via radial basis functions $[19,20]$. Namely, the norm of the inverse depends on the particular RBF, the dimension, and the minimal separation of the Hermite data, but not on the number or distribution of points. Indeed, the results in $\S 7$ contain one of the central estimates in [20] as a special case.

\section{Preliminaries}

Admissible spaces. We denote by $\mathscr{E}_{n}$ the set of all $C^{\infty}$ vector-valued functions $f: \mathbb{R}^{s} \rightarrow \mathbb{C}^{n}$, and by $\mathscr{E}_{n}^{\prime}$ the space of all $n$-component "column-vector" distributions compactly supported in $\mathbb{R}^{s}$. If $\phi \in \mathscr{E}_{n}^{\prime}$, then let $\phi(x)^{*}=\bar{\phi}(x)^{T}$ be the conjugate transpose of $\phi(x)$. The linear functional corresponding to the distribution $\phi(x)$ acts on $f \in \mathscr{E}_{n}$ via $\int_{\mathbb{R}^{s}} \phi(x)^{*} f(x) d^{s} x=\sum_{j=1}^{n} \int_{\mathbb{R}^{s}} \bar{\phi}_{j}(x) f_{j}(x) d^{s} x$, where the $f_{j}$ 's and $\phi_{j}$ 's are the components of $f$ and $\phi$.

Fourier transforms and convolutions will play an important role in what follows. As usual, we define the Fourier transform of a function or (tempered) distribution to be $\hat{f}(\xi):=\int_{\mathbb{R}^{s}} f(x) e^{-i x \cdot \xi} d^{s} x$. It is useful to note that compactly supported distributions are tempered and that the Fourier transform of a compactly supported distribution is an analytic function $[8$, p. 130;25, p. 307]. Let $\varphi$ be a scalar-valued distribution, and let $f \in \mathscr{E}_{n}$. We define 
the convolution $f * \varphi$ component-wise, with the $j$ th component being $[f * \varphi(x)]_{j}:=\int_{\mathbb{R}^{s}} f_{j}(x-y) \varphi(y) d^{s} y$. It follows from [25, p. 287] that $f * \varphi$ is in $\mathscr{E}_{n}$.

To incorporate side conditions, we will need to work with certain subspaces of $\mathscr{E}_{n}$, which we now define.

Definition 1.1. Let $\mathfrak{G}$ denote a subspace of $\mathscr{E}_{n}$ for which the following holds: If $g$ is in $\mathfrak{G}$ and $\varphi$ is an arbitrary scalar-valued distribution in $\mathscr{E}_{1}^{\prime}$, then $g * \varphi$ is in $\mathfrak{G}$. We shall call such spaces admissible.

Any admissible space $\mathfrak{G}$ is translation invariant. Let $\varphi_{x^{\prime}}(x)=\delta\left(x-x^{\prime}\right)$. If $g$ is in $\mathfrak{G}$ then so is $g\left(x-x^{\prime}\right)=g * \varphi_{x^{\prime}}$. Although we will not prove it here, one can show that a translation invariant subspace of $\mathscr{E}_{n}$ that is also closed in the $C^{\infty}$ topology is admissible.

For much of the paper, we will be concerned with admissible subspaces arising as kernels of constant-coefficient differential operators.

Definition 1.2. For an integer $\nu>0$, let $B_{1}(x), \ldots, B_{\nu}(x)$ be $\mathbb{C}^{n}$-valued polynomials defined on $\mathbb{R}^{s}$ and having degree $d$. Define the space

$$
\mathfrak{G}_{B}:=\left\{g \in \mathscr{E}_{n} \mid B_{j}(\partial)^{*} g \equiv 0, j=1, \ldots, \nu\right\}, \quad \text { where } \partial=\left(\partial_{x_{1}}, \ldots, \partial_{x_{s}}\right) \text {. }
$$

These spaces are admissible because $B_{j}(\partial)^{*}(g * \varphi)=\left(B_{j}(\partial)^{*} g\right) * \varphi=0$. (See [25, p. 287].)

If one takes all of the $B_{j}$ 's to be identically 0 , then one has the space $\mathscr{E}_{n}$. When $\nu=1$ and $n=3$, we may take $B_{1}(x)=\left(\begin{array}{lll}x_{1} & x_{2} & x_{3}\end{array}\right)^{T}$. For this case, $\mathfrak{G}_{B}$ is simply $\mathfrak{G}_{\text {div }}$. More generally, one can show that the integrability conditions required for a $p$-form $[7, \S 3.6]$ on $\mathbb{R}^{s}$ to be closed $[7$, p. 67] can be formulated using the coefficients of the $p$-form and an appropriate $\mathfrak{G}_{B}$.

G-linearly independent distributions. We suppose that data is generated by applying a finite number of compactly supported distributions to a function $f \in \mathscr{E}_{n}$. More precisely, let $\left\{\phi_{j}(x)\right\}_{j=1}^{N}$ be a linearly independent subset of $\mathscr{E}_{n}^{\prime}$, which is the space of $n$-component "column-vector" distributions compactly supported in $\mathbb{R}^{s}$, and assume that we are given data in the form

$$
\int_{\mathbb{R}^{s}} \phi_{j}(x)^{*} f(x) d^{s} x=d_{j}, \quad j=1, \ldots, N,
$$

where the $d_{j}$ 's are scalars and $\phi(x)^{*}=\overline{\phi(x)}^{T}$ is the conjugate transpose of $\phi(x)$.

Recall that when dealing with the example discussed in the introduction, we required that the distributions used be linearly independent when restricted to the space $\mathfrak{G}_{\mathrm{div}}$. This restriction is needed to avoid redundant data. In the general case, we will also require that the distributions that generate the data set must be compatible with the side conditions imposed. To accommodate this need for compatibility, we will say that the set $\left\{\phi_{j}(x)\right\}_{j=1}^{N}$ is $\mathfrak{G}$-linearly independent if the set $\left\{\left.\phi_{j}(x)\right|_{\mathcal{A}}\right\}_{j=1}^{N}$ is linearly independent.

Let $\pi_{m-1}^{s \rightarrow n}$ be all $\mathbb{C}^{n}$-valued polynomials on $\mathbb{R}^{s}$ having degree $m-1$ or less. To deal with interpolation problems requiring polynomial reproduction, we have to introduce the following subspaces of $\mathscr{E}_{n}^{\prime}$ :

$$
\mathscr{E}_{n, m}^{\prime}(\mathfrak{G}):=\left\{\phi \in \mathscr{E}_{n}^{\prime} \mid \int_{\mathbb{R}^{s}} \phi(x)^{*} p(x) d^{s} x=0 \text { for all } p \in \pi_{m-1}^{s \mapsto n} \cap \mathfrak{G}\right\},
$$


if $m \geq 1$. When $m=0$, we set $\mathscr{E}_{n, 0}^{\prime}(\mathfrak{G}):=\mathscr{E}_{n}^{\prime}$.

Several remarks are in order. When $\mathfrak{G}=\mathscr{E}_{n}$, we will write $\mathscr{E}_{n, m}^{\prime}$ for $\mathscr{E}_{n, m}^{\prime}(\mathfrak{G})$. From (1.2), note that if we have that $\phi \in \mathscr{E}_{n, m}^{\prime}$, then we also have $\phi \in \mathscr{E}_{n, m}^{\prime}(\mathfrak{G})$; consequently,

$$
\mathscr{E}_{n, m}^{\prime}(\mathfrak{G}) \supseteq \mathscr{E}_{n, m}^{\prime} .
$$

Finally, we note that the scalar-valued versions of these spaces were introduced by Madych and Nelson [16].

Matrix-valued $\mathfrak{G}$-CPDFs of order $m$. We now introduce the class of functions that will be used to generate our interpolants.

Definition 1.3. Let $h(x)$ be an $n \times n$ matrix whose components are in $C^{\infty}\left(\mathbb{R}^{s}\right)$ and whose columns are in an admissible space $\mathfrak{G}$. In addition, we require that $h(x)^{*}=h(-x)$ for all $x \in \mathbb{R}^{s}$. We will say that $h$ is an order- $m$ G-conditionally positive definite (G-CPD) $n \times n$ matrix-valued function if

$$
\int_{\mathbb{R}^{s}} \phi(x)^{*}(h * \phi)(x) d^{s} x \geq 0
$$

$$
\text { where }[h * \phi(x)]_{j}=\sum_{k=1}^{n} \int h_{j, k}(x-y) \phi_{k}(y) d^{s} y,
$$

for all $\phi \in \mathscr{E}_{n, m}^{\prime}(\mathfrak{G})$. Moreover, we will say that such an $h$ is strictly $\mathfrak{G}$-CPD if equality in (1.4) implies that $\int_{\mathbb{R}^{s}} \phi(x)^{*} g(x) d^{s} x=0$ for all $g \in \mathfrak{G}$.

Remark 1.4. We point out that when $m=0$, the distributions used in (1.4) are independent of $\mathfrak{G}$, and the only restriction on $h$ involving $\mathfrak{G}$ is that its columns belong to $\mathfrak{G}$. In case $\mathfrak{G}=\mathscr{E}_{n}$, we will write "CPD" rather than $\mathfrak{G}-\mathrm{CPD}$. Also, because $\mathscr{E}_{n, m}^{\prime}(\mathfrak{G}) \supseteq \mathscr{E}_{n, m}^{\prime}$, every order- $m$ G-CPD is also an order-m CPD.

All our interpolants will be of the form $h * \phi(x)+p(x)$, where $\phi$ will be assumed to be in $\mathscr{E}_{n, m}^{\prime}(\mathfrak{G})$ and $p(x)$ will be taken to be a $\mathbb{C}^{n}$-valued polynomial on $\mathbb{R}^{s}$ having degree $m-1$ or less-i.e., $p \in \pi_{m-1}^{s \mapsto n}$. Of course, precisely which $\phi$ and which $p$ should be chosen will depend on both the distributions generating the data set, on the data set itself, and on any side conditions one wishes to impose. Of course, when there are no side conditions, one may simply work in $\mathfrak{G}=\mathscr{E}_{n}$ itself.

Matrix-valued $\mathfrak{G}-\mathrm{CPD}$ functions generalize the notion of a scalar-valued CPD function, as defined in Gelfand and Vilenkin [9]. The definition that we have introduced here is directly related to the version of scalar-valued CPDFs worked out by Madych and Nelson [16]. The two formulations of scalar-valued CPDFs were shown to be equivalent [16]. Since the version in [16] fits our needs better than that in [9], we have based our own definition upon it. We will discuss examples of matrix-valued CPDFs in later sections, including ones that enable us to create "divergence-free" interpolants.

\section{Generalized Hermite interpolation Problems}

The problem of Hermite interpolation with polynomial reproduction is actually a family of problems, each of which depends on a nonnegative integer $m$ : 
Problem 2.1. Given a $\mathfrak{G}$-linearly independent set $\left\{\phi_{j}(x)\right\}_{j=1}^{N} \subset \mathscr{E}_{n}^{\prime}$, complex numbers $d_{j}, j=1, \ldots, N$, and an order- $m, n \times n$ matrix-valued $\mathfrak{G}$-CPD function $h$, and letting

$$
\mathscr{V}=\operatorname{Span}\left(\left\{\phi_{j}(x)\right\}_{j=1}^{N}\right) \quad \text { and } \quad \mathscr{W}=\mathscr{V} \cap \mathscr{E}_{n, m}^{\prime}(\mathfrak{G}),
$$

find $\psi \in \mathscr{W}$ and $p \in \pi_{m-1}^{s \mapsto n} \cap \mathfrak{G}$ such that $h * \psi \in \mathfrak{G}$ and

$$
\int_{\mathbb{R}^{s}} \phi_{j}(x)^{*}((h * \psi)(x)+p(x)) d^{s} x=d_{j},
$$

for $j=1, \ldots, N$. In addition, if for some polynomial $\tilde{p} \in \pi_{m-1}^{s \mapsto n} \cap \mathfrak{G}$ and for $j=1, \ldots, N$ each $d_{j}=\int_{\mathbb{R}^{s}} \phi_{j}(x)^{*} \tilde{p}(x) d^{s} x$, then we require that the only interpolant be $p(x)=\tilde{p}(x)$; that is, $\psi(x)=0$ above, and $p$ is unique.

It is clear that $\mathfrak{G}$-CPD matrix-valued functions are central to our formulation of the generalized Hermite interpolation problem described above. In particular, the condition on a $\mathfrak{G}-\mathrm{CPDF} h$ that $h(x)^{*}=h(-x)$ is important. Employing it, and standard results concerning convolution products involving distributions [25, p. 291], one obtains the following useful result, which will be used to show that certain interpolation matrices are selfadjoint.

Lemma 2.2. If $\phi$ and $\psi$ are in $\mathscr{E}_{n}^{\prime}$, and if $h$ is a $C^{\infty}, n \times n$-matrix-valued function that satisfies $h(x)^{*}=h(-x)$, then

$$
\overline{\int_{\mathbf{R}^{s}} \psi(x)^{*}(h * \phi)(x) d^{s} x}=\int_{\mathbb{R}^{s}} \phi(x)^{*}(h * \psi)(x) d^{s} x .
$$

Proof. Following [25], we let $\check{f}(x):=f(-x)$. We have

$$
\overline{\int_{\mathbb{R}^{s}} \psi(x)^{*}(h * \phi)(x) d^{s} x}=\sum_{j, k} \int_{\mathbb{R}^{s}} \psi_{j}(x)\left(\bar{h}_{j, k} * \bar{\phi}_{k}\right)(x) d^{s} x .
$$

It is a straightforward task to put the equation above into the form

$$
\overline{\int_{\mathbb{R}^{s}} \psi(x)^{*}(h * \phi)(x) d^{s} x}=\sum_{j, k} \int_{\mathbb{R}^{s}}\left(\check{\psi}_{j} * \bar{\phi}_{k}\right)(x) h_{k, j}(x) d^{s} x .
$$

By [25, Theorem 27.7, pp. 291-292], convolution is commutative for compactly supported distributions, and so $\check{\psi}_{j} * \bar{\phi}_{k}=\bar{\phi}_{k} * \check{\psi}_{j}$. Using this in the last equation and restoring matrix notation yields (2.2). Alternatively, one may use a Fourier transform approach based on a cutoff function and the fact that if $h$ has a Fourier transform $\hat{h}(\xi)$, then $\hat{h}(\xi)$ will be a selfadjoint matrix.

We will prove the following result, which will provide the framework for solving the class of interpolation problems described in Problem 2.1.

Theorem 2.3. With the notation of Problem 2.1, if $h$ is strictly $\mathfrak{G}-C P D$, and if the dimension of $\mathscr{V} / \mathscr{W}$ is equal to the dimension $m^{\prime}$ of $\pi_{m-1}^{s \rightarrow n} \cap \mathfrak{G}$, then Problem 2.1 is well poised.

Proof. Without loss of generality, we may assume that the distributions $\phi_{j}$, $j=1, \ldots, N-m^{\prime}$, form a basis for $\mathscr{W}$ and that $\mathscr{U}:=\operatorname{Span}\left(\left\{\phi_{j}\right\}_{j=N-m^{\prime}+1}^{N} \cong\right.$ $\mathscr{V} / \mathscr{W}$. (If this is not the case, simply pick a basis for $\mathscr{W}$ and complete it to a basis for $\mathscr{V}$ in the usual way. In carrying this out, one may have to use linear 
combinations of the $d_{j}$ 's.) Consequently, we can reformulate the first $N-m^{\prime}$ equations in (2.1) this way:

$$
\int_{\mathbb{R}^{s}} \phi_{j}(x)^{*}(h * \psi)(x) d^{s} x=d_{j}, \quad j=1, \ldots, N-m^{\prime} .
$$

If we expand $\psi \in \mathscr{W}$ in terms of the basis $\left\{\phi_{j}\right\}_{j=1}^{N-m^{\prime}}$, we get $\psi=\sum_{j=1}^{N-m^{\prime}} c_{j} \phi_{j}$. The equations above turn into the matrix equation $A c=d$, with $A_{j, k}=$ $\int_{\mathbf{R}^{s}} \phi_{j}(x)^{*}\left(h * \phi_{k}\right)(x) d^{s} x$. Note that Lemma 2.2 implies that $A_{j, k}=\bar{A}_{k, j}$, so $A$ is selfadjoint. In fact, we will show that $A$ is positive definite. Consider the quadratic form

$$
c^{*} A c=\int_{\mathbf{R}^{s}} \psi(x)^{*}(h * \psi)(x) d^{s} x, \quad \text { where } \psi=\sum_{j=1}^{N-m^{\prime}} c_{j} \phi_{j} \in \mathscr{E}_{n, m}^{\prime}(\mathfrak{G}) .
$$

From (1.4), we have that the right side of the equation above is nonnegative. But can $c^{*} A c=0$ ? For that to happen, we must have that $A c=0$. We would then have $\int_{\mathbb{R}^{s}} \psi(x)^{*}(h * \psi)(x) d^{s} x=0$, which, when put together with the fact that $h$ is a strictly $\mathfrak{G}$-CPD matrix-valued function, yields $\left.\psi\right|_{\mathfrak{B}}=0$. Since $\left\{\phi_{j}\right\}_{j=1}^{N}$ is $\mathfrak{G}$-linearly independent, the coefficients $c_{j}$ must all vanish. Thus, $c=0$ and $A$ is positive definite, and, consequently, invertible. Hence, we are able to uniquely solve (2.3) for $\psi$. Furthermore, as we remarked earlier, the fact that $h$ is a $\mathfrak{G}$-CPD matrix-valued function implies that $h * \psi$, which is a sum of columns of $h$ convolved with scalar-valued distributions, is in $\mathfrak{G}$.

There still remains the question of whether one can find a $p$ that satisfies the equations in (2.1) not covered in (2.3). Obviously, we can find $\psi$ so that the equations in (2.1) are solved for $j=1, \ldots, N-m^{\prime}$. The remaining equations have the form

$$
\int_{\mathbb{R}^{s}} \phi_{j}(x)^{*} p(x) d^{s} x=d_{j}^{\prime}, \quad j=N-m^{\prime}+1, \ldots, N,
$$

where $d_{j}^{\prime}=d_{j}-\int_{\mathbb{R}^{s}} \phi_{j}(x)^{*}(h * \psi)(x) d^{s} x, j=N-m^{\prime}, \ldots, N$, are known. Standard duality arguments and the assumption that $\operatorname{dim}(\mathscr{V} / \mathscr{W})=m^{\prime}=$ $\operatorname{dim}\left(\pi_{m-1}^{s \mapsto n} \cap \mathfrak{G}\right)$ can be employed to show that there is a unique $p \in \pi_{m-1}^{s \mapsto n} \cap \mathfrak{G}$ that solves the equations in (2.4).

We now state a proposition that was established in proving Theorem 2.3.

Proposition 2.4. Let $\left\{\phi_{j}\right\}_{j=1}^{M}$ be a $\mathfrak{G}$-linearly independent subset of the space $\mathscr{E}_{n, m}^{\prime}(\mathfrak{G})$. If $h$ is an order-m, $n \times n$ matrix-valued $\mathfrak{G}-C P D$ function, then the matrix $A$, with entries

$$
A_{j, k}=\int_{\mathbf{R}^{s}} \phi_{j}(x)^{*}\left(h * \phi_{k}\right)(x) d^{s} x,
$$

is selfadjoint and nonnegative. If $h$ is strictly $\mathfrak{G}-C P D$ as well, then the matrix $A$ is also positive definite.

Remark 2.5. Theorem 2.3 is directed at solving Problem 2.1. We want to point out that, at least in one other case, a straight interpolation problem involving no polynomial reproduction can be solved. Suppose that the data set is generated by $N-1$ distributions in $\mathscr{E}_{n, m}^{\prime}$, and by one other distribution that is merely 
in $\mathscr{E}_{n}^{\prime}$. As usual, label these $\phi_{j}$, with the one not in $\mathscr{E}_{n, m}^{\prime}$ being $\phi_{N}$. Let $h$ be strictly $\mathfrak{G}$-CPD. Form the matrix $A$ as in (2.5) above. If $A_{N, N}<0$, then $A$ is invertible.

To see this, observe that the matrix $A$ is selfadjoint. By Proposition 2.4, $A$ satisfies $c^{*} A c>0$ when the $N$ th component of $c$ is $0-$ i.e., $c$ is orthogonal to $e_{N}=(0, \ldots, 1)^{T}$. The Rayleigh-Ritz procedure then implies that $A$ has at least $N-1$ positive eigenvalues. On the other hand, $e_{N}^{*} A e_{N}<0$. A second application of the Rayleigh-Ritz procedure then shows that $A$ has at least one negative eigenvalue. Combining these statements, one sees that $A$ has $N-1$ positive eigenvalues and 1 negative eigenvalue, and so $A$ is invertible.

Although the situation described above seems quite specialized, it is not all that uncommon. For example, when $n=m=1$ and $\mathfrak{G}=\mathscr{E}_{1}$, the space $\pi_{0}^{s \mapsto 1} \cap \mathfrak{G}=\pi_{0}^{s \mapsto 1}$ is a one-dimensional space. The $N$-dimensional space of distributions generating the data set is either a subspace of $\mathscr{E}_{1,0}^{\prime}$ or it has a basis with $N-1$ distributions in $\mathscr{E}_{1,0}^{\prime}$ and one not in the space. $A$ will be invertible if that one distribution is such that $A_{N, N}<0$, something that happens in standard interpolation using the Hardy multiquadrics.

\section{Conditionally positive definite MATRIX-VAlued FUNCTIONS}

We now turn to a discussion of CPD $n \times n$ matrix-valued functions. This is the case where $\mathfrak{G}=\mathscr{E}_{n}$; there are no side conditions on the vector field that we want to interpolate. Treating this case will be helpful in dealing with the case in which $\mathfrak{G}$ is a proper subspace of $\mathscr{E}_{n}$. Indeed, by Remark 1.4 , an order$m$ G-CPD matrix-valued function is an order- $m$ CPD, so knowing a class of order- $m$ CPDFs is an important step in constructing a class of order- $m$ G-CPD matrix-valued functions.

The starting point for our discussion of matrix-valued CPDs is the MadychNelson version of the theory of order- $m$, continuous CPD scalar-valued functions; this is found in [16]. Concerning notation, we recall that when $\mathfrak{G}=\mathscr{E}_{n}$, the spaces $\pi_{m-1}^{s \mapsto n} \cap \mathfrak{G}=\pi_{m-1}^{s \mapsto n}$; the distributions in $\mathscr{E}_{n, m}^{\prime}$ annihilate all polynomials in $\pi_{m-1}^{s \mapsto n}$. Also, here and throughout the paper we will use standard multi-index notation; see $[8, \S 1.1]$. We are now ready to prove the result below, which will allow us to freely use Fourier transforms.

Proposition 3.1. If $h$ is a $C^{\infty}\left(\mathbb{R}^{s}\right), n \times n C P D$ matrix-valued function, then the entries of $h$ have polynomial growth, and therefore $h$ may be regarded as a tempered distribution. Moreover, $\hat{h}$, the Fourier transform of $h$, exists in the sense of tempered distributions.

Proof. Let $c \in \mathbb{C}^{n}$, and set $h_{c}(x):=c^{*} h(x) c$. It is clear that $h_{c}$ is $C^{\infty}$, and that

$$
\int_{\mathbb{R}^{s}} \bar{\phi}(x)\left(h_{c} * \phi\right)(x) d^{s} x=\int_{\mathbb{R}^{s}}(c \phi(x))^{*}[h *(c \phi)](x) d^{s} x \geq 0
$$

for every $C^{\infty}$ compactly supported function $\phi(x)$ in $\mathscr{E}_{1, m}^{\prime}$, because $c \phi \in$ $\mathscr{E}_{n, m}^{\prime}$. Since $\phi(x)$ is in both $C^{\infty}\left(\mathbb{R}^{s}\right)$ and $\mathscr{E}_{1, m}^{\prime}$, it satisfies the conditions required for $\phi$ to be in the space $\mathscr{D}_{m}$ [16, equation (2.10)], which comprises all compactly supported $C^{\infty}$ functions $\omega(x)$ such that

$$
\int_{\mathbb{R}^{s}} x^{\alpha} \omega(x) d^{s} x=0 \text { for all }|\alpha|<m .
$$


By the remark following (2.12) in [16], we have that $h_{c}$ is a continuous order- $m$ CPD function. When $m=0$, then $h_{c}$ is a (Bochner) positive definite function and is consequently bounded. When $m>0$, Corollary 2.3 in [16] implies that $h_{c}$ has polynomial growth. In either case, $h_{c}$ is seen to be a tempered distribution [25, p. 274]. In the usual way, one can write the entries in $h$ as linear combinations of $h_{c}$ 's with various $c$ 's:

$$
h_{j, k}=\frac{1}{2}\left\{h_{c}+i h_{\tilde{c}}-(1+i)\left(h_{e_{j}}+h_{e_{k}}\right)\right\}, \quad \text { where } c=e_{j}+e_{k} \text { and } \tilde{c}=e_{j}-i e_{k} \text {. }
$$

Consequently, the $h_{j, k}$ 's have polynomial growth and are therefore tempered distributions. That tempered distributions have Fourier transforms is well known $[8$, p. $98 ; 25$, p. 275$]$.

We wish to construct a class of CPD matrix-valued functions analogous to the scalar-valued CPD functions discussed in [16, §2]. The construction requires certain matrix-valued measures, which are special cases of the operator-valued measures discussed by Berberian [2, p. 6].

Let $\mathscr{B}$ be the Borel subsets of $\mathbb{R}^{s}$, and let $\mathscr{P}_{n}$ be the set of nonnegative definite $n \times n$ selfadjoint matrices. A function $\mu: \mathscr{B} \rightarrow \mathscr{P}_{n}$ that is additive and continuous will be termed an $n \times n$ selfadjoint, positive matrix-valued, Borel measure. We remark that if $c \in \mathbb{C}^{n}$, then $\mu_{c}(\cdot)=c^{*} \mu(\cdot) c$ defines a Borel measure on $\mathbb{R}^{s}$. As was the case for $h$, the entries of $\mu$ can be written as linear combinations of $\mu_{c}$ 's:

$$
\mu_{j, k}=\frac{1}{2}\left\{\mu_{c}+i \mu_{\tilde{c}}-(1+i)\left(\mu_{e_{j}}+\mu_{e_{k}}\right)\right\}, \quad \text { where } c=e_{j}+e_{k} \text { and } \tilde{c}=e_{j}-i e_{k} \text {. }
$$

The set of all such measures has a natural partial ordering. We will say that $\mu \geq \tilde{\mu}$ if for every $\Delta \in \mathscr{B}$ the difference $\mu(\Delta)-\tilde{\mu}(\Delta)$ is in $\mathscr{P}_{n}$. Because measures are often given in "differential form," we will also write $d \mu \geq d \tilde{\mu}$ to mean $\mu \geq \tilde{\mu}$. We are now ready to state our next result.

Theorem 3.2. Let $d \mu(\xi)$ be an $n \times n$ selfadjoint, positive matrix-valued, Borel measure defined on $\mathbb{R}^{s} \backslash\{0\}$, and suppose that $\int_{\|\xi\| \leq 1}|\xi|^{j} d \mu(\xi)$ is finite for all $j \geq 2 m$, and also that $\int_{\|\xi\| \geq 1}|\xi|^{k} d \mu(\xi)$ is finite for all $k$. In addition, let $\left\{a_{\gamma}\right\}_{|\gamma| \leq 2 m}$ be a set of $n \times n$ selfadjoint matrices labeled by the multi-index $\gamma$, and satisfying the condition that for every finite subset $\left\{c_{\gamma}\right\}_{|\gamma| \leq m}$ in $\mathbb{C}^{n}$

$$
\sum_{|\alpha|=m|\beta|=m} \sum_{\beta}^{*} a_{\alpha+\beta} c_{\alpha} \geq 0
$$

Then,

$$
h(x):=\int_{\mathbb{R}^{s}}\left[e^{i x \cdot \xi}-\sum_{r=0}^{2 m-1} \frac{(i x \cdot \xi)^{r}}{r !}\right] d \mu(\xi)+\sum_{|\gamma| \leq 2 m} a_{\gamma} \frac{(i x)^{\gamma}}{\gamma !}
$$

is a CPD $n \times n$ matrix-valued function.

Proof. It is clear that $h(x)^{*}=h(-x)$. Because $d \mu$ decays fast enough to support all powers of $|\xi|$, one may freely differentiate under the integral sign, so $h$ is infinitely differentiable. Let $\phi \in \mathscr{E}_{n, m}^{\prime}$, and suppose that in addition $\phi$ is $C^{\infty}$. (In the notation of [16, equation (2.10)], each component of $\phi$, namely $\phi_{j}$, is in $\mathscr{D}_{m}$, the space of all compactly supported $C^{\infty}$ functions 
whose power moments vanish to order $m-1$.) A straightforward application of Fubini's theorem yields

$$
\begin{aligned}
\int_{\mathbf{R}^{s}} \phi(x)^{*}(h * \phi)(x) d^{s} x \\
=\int_{\mathbb{R}^{s}} \hat{\phi}(\xi)^{*} d \mu(\xi) \hat{\phi}(\xi) \\
\quad-\sum_{r=0}^{2 m-1} \int_{\mathbb{R}^{s}} \sum_{j, k=1}^{n}\left\{\int_{\mathbb{R}^{s}} \int_{\mathbb{R}^{s}} \bar{\phi}_{j}(x) \frac{[i(x-y) \cdot \xi]^{r}}{r !} \phi_{j}(y) d^{s} y d^{s} x\right\} d \mu_{j, k}(\xi) \\
\quad+\sum_{|\gamma| \leq 2 m} \sum_{j, k=1}^{n} a_{\gamma}^{j, k}\left\{\int_{\mathbb{R}^{s}} \int_{\mathbb{R}^{s}} \bar{\phi}_{j}(x) \frac{[i(x-y)]^{\gamma}}{\gamma !} \phi_{k}(y) d^{s} y d^{s} x\right\} .
\end{aligned}
$$

Because $\phi \in \mathscr{E}_{n, m}^{\prime}$, all integrals of the form $\int_{\mathbb{R}^{s}} y^{\gamma} \phi_{k}(y) d^{s} y$ or $\int_{\mathbb{R}^{s}} x^{\gamma} \bar{\phi}_{j}(x) d^{s} x$ vanish. Taking this into account in the last equation, and using

$$
(-i)^{|\gamma|} \int_{\mathbb{R}^{s}} x^{\gamma} f(x) d^{s} x=\partial^{\gamma} \hat{f}(0),
$$

we see that

$$
\begin{aligned}
\int_{\mathbb{R}^{s}} \phi(x)^{*}(h * \phi)(x) d^{s} x \\
=\int_{\mathbb{R}^{s}} \hat{\phi}(\xi)^{*} d \mu(\xi) \hat{\phi}(\xi) \\
\quad+\sum_{|\alpha|=m|\beta|=m} \sum_{j, k=1}^{n} a_{\alpha+\beta}^{j, k}\left\{\int_{\mathbb{R}^{s}} \bar{\phi}_{j}(x) \frac{(i x)^{\alpha}}{\alpha !} d^{s} x \int_{\mathbb{R}^{s}} \frac{(-i y)^{\beta}}{\beta !} \phi_{k}(y) d^{s} y\right\} \\
=\int_{\mathbb{R}^{s}} \hat{\phi}(\xi)^{*} d \mu(\xi) \hat{\phi}(\xi)+\sum_{|\alpha|=m|\beta|=m} \sum_{3}\left\{\frac{\partial^{\alpha} \hat{\phi}(0)}{\alpha !}\right\}^{*} a_{\alpha+\beta}\left\{\frac{\partial^{\beta} \hat{\phi}(0)}{\beta !}\right\} .
\end{aligned}
$$

We remark that the $C^{\infty}$ distributions in $\mathscr{E}_{n, m}^{\prime}$ are dense in that space. Consequently, taking limits in the last equation implies that (3.3) holds for all $\phi$ in $\mathscr{E}_{n, m}^{\prime}$. From our assumptions on $d \mu$ and $a_{\gamma}$, it is also clear that the right side above is nonnegative and that $h$ is a matrix-valued CPD function.

Notice that in (3.2) we have " $i x$ " rather than " $-i x$," which is what appears in the scalar case treated in [16]. The reason for this is that, to accommodate the need for matrix multiplication, our definition of matrix-valued CPD uses convolution in a slightly different way than in [16] or [9]. In the scalar case, it is easy to show that the definitions are equivalent. In fact, if $h(x)$ is a matrix-valued CPDF, one can show that $h(-x)$ is also a CPDF.

The form of the matrix-valued CPDF given in (3.2) will be used as a guide in constructing matrix-valued $\mathfrak{G}$-CPDFs for the spaces $\mathfrak{G}_{B}$ introduced in $\S 1$.

\section{A CHARACTERIZATION OF CERTAIN COMPACTLY SUPPORTED DISTRIBUTIONS}

Recall that if $B_{1}, \ldots, B_{\nu}$ are in $\pi_{d}^{s \mapsto n}$, then we define $\mathfrak{G}_{B}$ via

$$
\begin{aligned}
\mathfrak{G}_{B}:=\left\{g \in \mathscr{C}_{n} \mid B_{j}(\partial)^{*} g \equiv 0, j=1\right. & 1, \ldots, \nu\}, \\
& \text { where } \partial=\left(\partial_{x_{1}}, \ldots, \partial_{x_{s}}\right) .
\end{aligned}
$$


In order to construct examples of matrix-valued $\mathfrak{G}$-CPDFs when the admissible space has the form (4.1), we need to characterize the compactly supported distributions in $\mathscr{E}_{n, m}^{\prime}\left(\mathfrak{G}_{B}\right)$.

Theorem 4.1. A compactly supported distribution $\phi$ is in $\mathscr{E}_{n, m}^{\prime}\left(\mathfrak{G}_{B}\right)$ if and only if there are compactly supported, scalar-valued distributions $\sigma_{1}(x), \cdots, \sigma_{\nu}(x)$ belonging to $\mathscr{E}_{1}^{\prime}$ and a compactly supported distribution $\phi_{m} \in \mathscr{E}_{n, m}^{\prime}\left(\mathscr{E}_{n}\right):=\mathscr{E}_{n, m}^{\prime}$ such that $\hat{\phi}$, the Fourier transform of $\phi$, has the form

$$
\hat{\phi}(\xi)=\sum_{j=1}^{\nu} \hat{\sigma}_{j}(\xi) B_{j}(-i \xi)+\hat{\phi}_{m}(\xi) .
$$

Proof. It is straightforward to show that if $p$ and $q$ are in $\pi_{m-1}^{s \mapsto n}$, then the sesquilinear form

$$
\langle p, q\rangle:=\left[q\left(\partial_{x}\right)\right]^{*} p(0)=\sum_{|\alpha| \leq m-1} \frac{\left[\partial^{\alpha} q(0)\right]^{*} \partial^{\alpha} p(0)}{\alpha !}
$$

defines an inner product on $\pi_{m-1}^{s \mapsto n}$. Let $f$ be a $C^{\infty}$ function, and let $T_{m-1}[f]$ be the order- $(m-1)$ Taylor polynomial for $f$. From (4.1), it easily follows that $p$ is in $\pi_{m-1}^{s \mapsto n} \cap \mathfrak{G}_{B}$ if and only if, for $j=1, \ldots, \nu$ and $|\alpha| \leq m-1$,

$$
\left\langle p, B_{j, \alpha}\right\rangle=0, \quad \text { where } B_{j, \alpha}(x):=T_{m-1}\left[x^{\alpha} B_{j}(x)\right] .
$$

Thus, we have that

$$
p \in \pi_{m-1}^{s \mapsto n} \cap \mathfrak{G}_{B} \quad \text { if and only if } \quad p \in\left\{B_{j, \alpha}\right\}^{\perp} .
$$

On the other hand, if $\phi$ is in $\mathscr{E}_{n, m}^{\prime}\left(\mathfrak{G}_{B}\right)$, then we have

$$
\int_{\mathbb{R}^{s}} \phi^{*} p d^{s} x=\sum_{\alpha}\left(\int_{\mathbb{R}^{s}} \phi^{*} x^{\alpha} d^{s} x\right) \frac{\partial^{\alpha} p(0)}{\alpha !}=0
$$

Since $(-i)^{|\gamma|} \int_{\mathbb{R}^{s}} x^{\gamma} \phi(x) d^{s} x=\partial^{\gamma} \hat{\phi}(0)$, we can rewrite the left side of the last equation as

$$
\int_{\mathbb{R}^{s}} \phi^{*} p d^{s} x=\sum_{\alpha} \frac{\left[i^{|\gamma|} \partial^{\gamma} \hat{\phi}(0)\right]^{*} \partial^{\alpha} p(0)}{\alpha !}=:\left\langle p(x), T_{m-1}[\hat{\phi}(i x)]\right\rangle,
$$

from which it follows that $\phi \in \mathscr{E}_{n, m}^{\prime}\left(\mathfrak{G}_{B}\right)$ if and only if $\left\langle p(x), T_{m-1}[\hat{\phi}(i x)]\right\rangle=0$ for all $p \in \pi_{m-1}^{s \mapsto n} \cap \mathfrak{G}$. The upshot is that $T_{m-1}[\hat{\phi}(i x)]$ is orthogonal to the whole space, $\pi_{m-1}^{s \mapsto n} \cap \mathfrak{G}_{B}=\left\{B_{j, \alpha}\right\}^{\perp}$. Consequently, $T_{m-1}[\hat{\phi}(i x)] \in \operatorname{Span}\left(B_{j, \alpha}\right)$, and so we can find constants $\frac{(-i)^{|\alpha|}}{\alpha !} c_{j, \alpha}$ such that

$$
T_{m-1}[\hat{\phi}(i x)]=\sum_{j=1}^{\nu} \sum_{|\alpha| \leq m-1} \frac{(-i)^{|\alpha|}}{\alpha !} c_{j, \alpha} B_{j, \alpha}(x) .
$$

Using (4.4), we can rewrite this as

$$
T_{m-1}[\hat{\phi}(i x)]=T_{m-1}\left[\sum_{j=1}^{\nu}\left(\sum_{|\alpha| \leq m-1} \frac{(-i)^{|\alpha|}}{\alpha !} c_{j, \alpha} x^{\alpha}\right) B_{j}(x)\right] .
$$


Let $\hat{\sigma}_{j}$ be the Fourier transform of a compactly supported distribution that satisfies the condition that $\partial_{\xi}^{\alpha} \hat{\sigma}_{j}(0)=c_{j, \alpha},|\alpha| \leq m-1$. The existence of such distributions follows from standard arguments; see [9]. Inserting $\hat{\sigma}_{j}$ as a replacement for the polynomial expression in large parentheses yields $T_{m-1}[\hat{\phi}(i x)]=$ $T_{m-1}\left[\sum_{j=1}^{\nu} \hat{\sigma}_{j}(-i x) B_{j}(x)\right]$. From this, we see that $\phi$ is in $\mathscr{E}_{n, m}^{\prime}\left(\mathfrak{G}_{B}\right)$ if and only if

$$
T_{m-1}\left[\hat{\phi}(\xi)-\sum_{j} \hat{\sigma}_{j}(\xi) B_{j}(-i \xi)\right]=0 .
$$

Set $\phi_{m}(x)=\phi(x)-\sum_{j} B_{j}\left(-\partial_{x}\right) \sigma_{j}(x)$. It is clear that $\phi_{m}$ is a compactly supported distribution on $\mathscr{E}_{n}$, that $\hat{\phi}_{m}(\xi)=\hat{\phi}(\xi)-\sum_{j} \hat{\sigma}_{j}(\xi) B_{j}(-i \xi)$, and, from (4.6), that $T_{m-1}\left[\hat{\phi}_{m}(\xi)\right]=0$. This last equation implies that $\phi_{m}$ is in $\mathscr{E}_{n, m}^{\prime}$. Thus, $\phi$ is in $\mathscr{E}_{n, m}^{\prime}\left(\mathfrak{G}_{B}\right)$ if and only if it has the form (4.2).

In the course of proving the last result, we produced a characterization of all polynomials in $\pi_{m-1}^{s \mapsto n} \cap \mathfrak{G}_{B}$. This characterization is given in (4.5) above and is restated in the corollary below for future reference.

Corollary 4.2. Let $\mathfrak{G}_{B}$ be as in (4.1), let $T_{m-1}[f]$ be the $(m-1)$-degree Taylor polynomial for an analytic function $f$, and let $\pi_{m-1}^{s \mapsto n}$ be endowed with the inner product (4.3). A polynomial $p$ is in $\pi_{m-1}^{s \leftrightarrow n} \cap \mathfrak{G}_{B}$ if and only if $p \in\left\{T_{m-1}\left[x^{\alpha} B_{j}(x)\right]\right\}^{\perp}$.

In addition to the result stated in Corollary 4.2, the proof of Theorem 4.1 provides us with a simple but useful fact concerning the distributions constructed there.

Corollary 4.3. Let $\mathfrak{G}_{B}$ be as in (4.1). With the notation of Theorem 4.1, the compactly supported distribution $\phi_{B}(x):=\sum_{j} B_{j}\left(-\partial_{x}\right) \sigma_{j}(x)$ satisfies $\int_{\mathbf{R}^{s}} \phi_{B}(x)^{*} g(x) d^{s} x=0$ for all $g \in \mathfrak{G}_{B}$.

Proof. By breaking $\phi_{B}$ and $g$ into their components and integrating by parts, one sees that

$$
\int_{\mathbf{R}^{s}} \phi_{B}(x)^{*} g(x) d^{s} x=\sum_{j=1}^{\nu} \int_{\mathbb{R}^{s}} \sigma_{j}(x) B_{j}\left(\partial_{x}\right)^{*} g(x) d^{s} x .
$$

From (4.1), each term in the sum on the right above vanishes.

\section{G-CONDITIONALLY POSITIVE DEFINITE MATRIX-VALUED FUNCTIONS}

We wish to explore two questions that concern the class of admissible subspaces, $\mathfrak{G}_{B}$. First, which matrix-valued CPDFs having the form (3.2) are actually $\mathfrak{G}$-CPDFs? Second, under what conditions will they be strictly $\mathfrak{G}-\mathrm{CPD}$ ? We will discuss the first question in this section, and the second in the next.

The result that follows is a surprisingly simple condition for a CPDF to be a $\mathfrak{G}-\mathrm{CPDF}$, provided of course that the admissible space is $\mathfrak{G}_{B}$.

Theorem 5.1. Let $\mathfrak{G}_{B}$ be as in (4.1). If $h(x)$ is a CPD matrix-valued function and if the columns of $h$ are in $\mathfrak{G}_{B}$, then $h$ is a $\mathfrak{G}-C P D$ matrix-valued function. 
Proof. By Theorem 4.1, one has that every $\phi \in \mathscr{E}_{n, m}^{\prime}\left(\mathfrak{G}_{B}\right)$ can be split into the sum

$$
\phi=\phi_{B}+\phi_{m}
$$

where $\phi_{m} \in \mathscr{E}_{n, m}^{\prime}$ and $\phi_{B}$ is as in Corollary 4.3. Using Lemma 2.2 and (5.1), one has

$$
\begin{aligned}
\int_{\mathbb{R}^{s}} \phi(x)^{*} h * \phi(x) d^{s} x \\
=\int_{\mathbb{R}^{s}} \phi_{m}(x)^{*} h * \phi_{m}(x) d^{s} x \\
\quad+\int_{\mathbb{R}^{s}} \phi_{B}(x)^{*} h * \phi_{B}(x) d^{s} x+2 \mathfrak{R}\left\{\int_{\mathbb{R}^{s}} \phi_{B}(x)^{*} h * \phi_{m}(x) d^{s} x\right\} .
\end{aligned}
$$

Since $h * \phi=\sum_{k} h^{k} * \phi_{k}$ is the sum of convolutions of functions in $\mathfrak{G}_{B}$ with compactly supported distributions (the columns $h^{k}$ are assumed to be in $\mathfrak{G}_{B}$ ), $h * \phi$ is in $\mathfrak{G}$. Consequently, we see from Corollary 4.3 that $\int_{\mathbf{R}^{s}} \phi_{B}(x)^{*} h *$ $\phi_{m}(x) d^{s} x=0$. Thus, in (5.2) all the terms containing $\phi_{B}$ vanish, and so (5.2) becomes

$$
\int_{\mathbb{R}^{s}} \phi(x)^{*} h * \phi(x) d^{s} x=\int_{\mathbb{R}^{s}} \phi_{m}(x)^{*} h * \phi_{m}(x) d^{s} x .
$$

Finally, by the fact that $h$ is an order- $m$ CPD, the right side of (5.3) is nonnegative, which implies that $h$ is a $\mathfrak{G}-\mathrm{CPDF}$.

As it stands, the theorem above does not provide a means for constructing $\mathfrak{G}-\mathrm{CPD}$ matrix-valued functions. However, we may combine it with the form for a matrix-valued CPDF given in Theorem 3.2 to obtain explicit expressions for such functions.

Corollary 5.2. Adopt the notation and assumptions listed in Theorem 3.2 , let $\mathfrak{G}_{B}$ be as in (4.1), and suppose that $B_{j}$ satisfies

$$
B_{j}(\partial)^{*}\left\{\sum_{r=0}^{2 m-1} \frac{(i x \cdot \xi)^{r}}{r !}\right\}=\sum_{k=1}^{\nu} \mathfrak{p}_{k}(x, \xi) B_{k}(-i \xi)^{*},
$$

whenever $m \geq 1$. Here, $\mathfrak{p}_{k}(x, \xi)$ is a polynomial in $x$ and $\xi$. If the positive, $n \times n$ matrix-valued measure $d \mu(\xi)$ satisfies $B_{j}(-i \xi)^{*} d \mu(\xi) \equiv 0$ for $j=1, \ldots, \nu$, and if, in addition, the columns of the matrix-valued polynomial $h_{2 m}(x):=\sum_{|\gamma| \leq 2 m} a_{\gamma}(i x)^{\gamma} /(\gamma !)$ are in $\mathfrak{G}$, then $h(x)$ is a $\mathfrak{G}$-CPD matrix-valued function.

Proof. Note that the measure in (3.2) decays fast enough at infinity for us to interchange the integral in (3.2) and any derivative. Using this fact, (5.4), and the assumption that the columns of $h_{2 m}$ are in $\mathfrak{G}_{B}$, we have that, for $j=1, \ldots, \nu$,

$$
\begin{aligned}
B_{j}(\partial)^{*} h(x) & =\int_{\mathbf{R}^{s}} B_{j}\left(\partial_{x}\right)^{*}\left[e^{i x \cdot \xi}-\sum_{r=0}^{2 m-1} \frac{(i x \cdot \xi)^{r}}{r !}\right] d \mu(\xi)+B_{j}(\partial)^{*} h_{2 m}(x) \\
& =\int_{\mathbb{R}^{s}}\left[e^{i x \cdot \xi} B_{j}(-i \xi)^{*}-\sum_{k=1}^{\nu} \mathfrak{p}_{k}(x, \xi) B_{k}(-i \xi)^{*}\right] d \mu(\xi) .
\end{aligned}
$$


Since $B_{j}(-i \xi)^{*} d \mu(\xi) \equiv 0$, the right side of the last equation is identically 0 . Consequently, $B_{j}(\partial)^{*} h(x) \equiv 0$ for $j=1, \ldots, \nu$, and so $h \in \mathfrak{G}$. From Theorem 5.1, $h$ is a $\mathfrak{G}-\mathrm{CPD}$ matrix-valued function.

Remark 5.3. When $m=0$, then (5.4) is vacuous, and there are no restrictions on the $B_{j}$ 's. Probably the most useful criterion for (5.4) holding for general $m \geq 1$ is the following: If each $B_{j}$ is a homogeneous polynomial, then (5.4) holds. Consequently, Corollary 5.2 applies for such a set of $B_{j}$ 's.

Proof. To see this, observe that for any single-variable polynomial $\mathfrak{p}$, the chain rule implies that $\partial_{x}^{\alpha} \mathfrak{p}(i x \cdot \xi)=\mathfrak{p}^{(|\alpha|)}(i x \cdot \xi)(i \xi)^{\alpha}$, where $\mathfrak{p}^{(l)}$ is the lth derivative of $\mathfrak{p}$. Since a homogeneous polynomial in $\partial_{x}$ is a linear combination of terms of the form $\partial_{x}^{\alpha}$ with $|\alpha|$ being fixed, and since the $B_{j}$ 's are homogeneous, we have $B_{j}(\partial)^{*} \mathfrak{p}(i x \cdot \xi)=\mathfrak{p}^{(l)}(i x \cdot \xi) B_{j}(-i \xi)^{*}$, from which (5.4) follows immediately.

\section{STRICTLY $\mathfrak{G}$-CONDITIONALly POSITIVE DEFINITE MATRIX-VALUED FUNCTIONS}

Earlier, we posed two questions, the first of which concerned which CPDFs of the form (3.2) were $\mathfrak{G}-C P D F s$ when the admissible space was $\mathfrak{G}_{B}$. This we feel that we have satisfactorily answered with the last three results. We now wish to address the second question, which concerns the issue of when a $\mathfrak{G}-\mathrm{CPD}$ matrix-valued function is strictly $\mathfrak{G}-\mathrm{CPD}$.

To do this, we need to deal with notation and terminology. First, recall that we write $d \mu \geq d \tilde{\mu}$ for matrix-valued measures, provided $\mu(\Delta)-\tilde{\mu}(\Delta)$ is a nonnegative definite matrix for every Borel set $\Delta \subset \mathbb{R}^{s}$. Second, let $\mathscr{S}_{n}$ denote the space of Schwartz functions $[8,25]$ in $\mathscr{E}_{n}$. We will say that the intersection $\mathscr{S}_{n} \cap \mathfrak{G}_{B}$ is weakly sequentially dense in $\mathfrak{G}_{B}$ if for every $g$ in $\mathfrak{G}_{B}$ there is a sequence of functions $g_{k} \in \mathscr{S}_{n} \cap \mathfrak{G}_{B}$ such that, for every $\phi \in \mathscr{E}_{n}^{\prime}$,

$$
\lim _{k \rightarrow \infty} \int_{\mathbb{R}^{s}} \phi^{*}(x) g_{k}(x) d^{s} x=\int_{\mathbb{R}^{s}} \phi^{*}(x) g(x) d^{s} x .
$$

For each fixed $\xi \in \mathbb{R}^{s}$, let $\Pi(\xi)$ be the orthogonal projection (relative to $\mathbb{C}^{n}$ ) onto $\left\{B_{1}(-i \xi), \ldots, B_{\nu}(-i \xi)\right\}^{\perp}$. We have the following result.

Theorem 6.1. Let $\mathfrak{G}_{B}$ and $h$ satisfy the assumptions of Corollary 5.2, and let $\mathfrak{G}_{B} \cap \mathscr{S}_{n}$ be weakly sequentially dense in $\mathfrak{G}_{B}$. In addition, assume that the rank of $\Pi(\xi)$ vanishes only on sets of Lebesgue measure 0. If the positive matrix-valued Borel measure $d \mu(\xi)$ in (3.2) satisfies

$$
d \mu(\xi) \geq \Pi(\xi) d \eta(\xi)
$$

where $d \eta$ is a positive scalar-valued Borel measure that has support containing an open subset of $\mathbb{R}^{s}$, then $h$ is a strictly $\mathfrak{G}-C P D$ matrix-valued function.

Proof. Since $h$ satisfies the conditions of Corollary 5.2, $h$ is a $\mathfrak{G}-\mathrm{CPDF}$. From the proof of Theorem 5.1, we recall that every $\phi \in \mathscr{E}_{n, m}^{\prime}\left(\mathfrak{G}_{B}\right)$ has the decomposition given in (5.1), and that $\int_{\mathbb{R}^{s}} \phi(x)^{*} h * \phi(x) d^{s} x$ satisfies (5.3), where $\phi_{m} \in \mathscr{E}_{n, m}^{\prime}$. Since $h$ is of the form (3.2), we have that (3.3) holds with $\phi$ replaced by $\phi_{m}$. By the assumptions on the polynomial part in (3.3), the associated quadratic form is nonnegative. Putting all of this together, we arrive at 
this inequality:

$$
\begin{aligned}
\int_{\mathbb{R}^{s}} \phi(x)^{*} h * \phi(x) d^{s} x & =\int_{\mathbb{R}^{s}} \phi_{m}(x)^{*} h * \phi_{m}(x) d^{s} x \\
& \geq \int_{\mathbb{R}^{s}} \hat{\phi}_{m}(\xi)^{*} d \mu(\xi) \hat{\phi}_{m}(\xi) .
\end{aligned}
$$

Combining (6.1) with (6.2) yields

$$
\int_{\mathbb{R}^{s}} \hat{\phi}(\xi)^{*} d \mu(\xi) \hat{\phi}(\xi) \geq \int_{\mathbb{R}^{s}} \hat{\phi}_{m}(\xi)^{*} \Pi(\xi) \hat{\phi}_{m}(\xi) d \eta(\xi) .
$$

To show that $h$ is strictly $C P D$, we need to show that if the left side of (6.3) vanishes, then $\left.\phi\right|_{\mathfrak{G}_{B}}=0$. Since $\phi=\phi_{B}+\phi_{m}$, and since $\left.\phi_{B}\right|_{\mathfrak{G}_{B}}=0$, we need only show that $\left.\phi_{m}\right|_{\mathfrak{G}_{B}}=0$. Clearly, if the left side of (6.3) is 0 , then we have that the right side, which is nonnegative, is also 0 ; that is, we have that

$$
\int_{\mathbb{R}^{s}} \hat{\phi}_{m}(\xi)^{*} \Pi(\xi) \hat{\phi}_{m}(\xi) d \eta(\xi)=0 .
$$

Since the support of $d \eta$ includes an open set in $\mathbb{R}^{s}$, and since $\hat{\phi}_{m}(\xi)^{*} \Pi(\xi) \hat{\phi}_{m}(\xi)$ $\geq 0$ for every $\xi \in \mathbb{R}^{s}$, we have that

$$
\hat{\phi}_{m}(\xi)^{*} \Pi(\xi) \hat{\phi}_{m}(\xi)=0
$$

on some open subset $\mathscr{N}$ of $\mathbb{R}^{s}$. This and the fact that $\Pi$ is an orthogonal projection imply that

$$
\Pi(\xi) \hat{\phi}_{m}(\xi)=0 \text { for all } \xi \in \mathcal{N},
$$

and so, on $\mathscr{N}, \phi_{m}(\xi)$ is in the span of the $B_{j}(-i \xi)$ 's. Pick a point $\xi_{0}$ in $\mathscr{N}$ at which the dimension of $\operatorname{Span}\left\{B_{1}(-i \xi), \ldots, B_{\nu}(-i \xi)\right\}$ is a maximum, $\nu_{0}$. At $\xi_{0}$, one may select a basis for the span of the $B_{j}\left(-i \xi_{0}\right)$ 's. After relabeling (if necessary), we take this basis to be $\left\{B_{1}\left(-i \xi_{0}\right), \ldots, B_{\nu_{0}}\left(-i \xi_{0}\right)\right\}$. Recall that this happens if and only if a $\nu_{0} \times \nu_{0}$ subdeterminant of the matrix with columns $B_{1}\left(-i \xi_{0}\right), \ldots, B_{\nu_{0}}\left(-i \xi_{0}\right)$ is not 0 . A straightforward continuity argument shows that, for all $\xi$ 's in a small neighborhood of $\xi_{0}$, the same subdeterminant of the matrix with columns $B_{1}(-i \xi), \ldots, B_{\nu_{0}}(-i \xi)$ will be nonvanishing. Thus, in that small neighborhood of $\xi_{0}$ the set $\left\{B_{1}(-i \xi), \ldots, B_{\nu_{0}}(-i \xi)\right\}$ is a basis for the span of all the $B_{j}$ 's. We now restrict our attention to the intersection of the small neighborhood of $\xi_{0}$ with $\mathscr{N}$. Indeed, by choosing $\mathscr{N}$ sufficiently small to begin with, we may assume that this intersection coincides with $\mathcal{N}$.

Cramer's rule can be used to show that on $\mathscr{N}$ we have

$$
\hat{\phi}_{m}(\xi)=\sum_{j=1}^{\nu_{0}} \frac{\tau_{j}(\xi)}{\partial(\xi)} B_{j}(-i \xi),
$$

where $\mathfrak{d}(\xi)$ is a polynomial in $\xi$, and the $\tau_{j}$ 's are linear combinations of polynomials multiplying the components of $\hat{\phi}_{m}(\xi)$. Since these components are entire functions in $\xi$, the $\tau_{j}(\xi)$ 's are also entire functions in $\xi$. Multiplying both sides of $(6.6)$ by $\mathfrak{d}(\xi)$ gives

$$
\mathfrak{d}(\xi) \hat{\phi}_{m}(\xi)=\sum_{j=1}^{\nu_{0}} \tau_{j}(\xi) B_{j}(-i \xi) \quad \text { for all } \xi \in \mathscr{N}
$$


Both sides of (6.7) are entire functions in $\xi$; their agreeing on $\mathscr{N}$ is sufficient to guarantee that they are equal for all $\xi$ in $\mathbb{C}^{s}$, and of course in $\mathbb{R}^{s}$. Since $\mathfrak{d}(\xi)$ is a polynomial that is not identically 0 , it can only vanish on sets of Lebesgue measure 0 . Consequently, (6.6) holds except for sets of Lebesgue measure 0 .

Simple Fourier transform manipulations imply that any Schwartz function $g$ will be in $\mathfrak{G}_{B}$ if and only if $B_{j}(-i \xi)^{*} \hat{g}(\xi) \equiv 0$ for all $\xi \in \mathbb{R}^{s}$. Using this and the fact that $(6.6)$ holds for almost every $\xi$, we get

$$
\hat{\phi}_{m}(\xi)^{*} \hat{g}(\xi)=0 \text {, }
$$

except on sets of Lebesgue measure 0 . Integrating both sides and using Parseval's relation-really, the definition of the Fourier transform of a distribution in this case-, we obtain

$$
\int_{\mathbb{R}^{s}} \phi_{m}(x)^{*} g(x) d^{s} x=0 \text { for all } g \in \mathfrak{G} \cap \mathscr{S}_{n} .
$$

Using the fact that $\mathfrak{G}_{B} \cap \mathscr{S}_{n}$ is weakly sequentially dense in $\mathfrak{G}_{B}$, one may easily show that (6.8) holds for all $g \in \mathfrak{G}_{B}$. It therefore follows that $\left.\phi_{m}\right|_{\mathfrak{G}_{B}}=0$, which is precisely what we needed in order to prove that $h$ is strictly $\mathfrak{G}-\mathrm{CPD}$.

Remark 6.2. The admissible spaces $\mathscr{E}_{n}$ and $\mathfrak{G}_{\text {div }}$ satisfy the conditions of Theorem 6.1. First, each of these spaces is generated by homogeneous polynomials. In the case of $\mathscr{E}_{n}$, these may be taken to be identically 0 . For the space $\mathfrak{G}_{\text {div }}$ the polynomial is linear. By Remark 5.3, any admissible $\mathfrak{G}_{B}$ with the $B_{j}$ 's being homogeneous satisfies the part of Theorem 6.1 that requires that the conditions of Corollary 5.2 be satisfied. Second, in all of them $\mathscr{S}_{n} \cap \mathfrak{G}_{B}$ is weakly sequentially dense. For $\mathfrak{G}_{B}=\mathscr{E}_{n}$, the compactly supported $C^{\infty}$ functions are obviously dense in $\mathscr{E}_{n}$ in the topology of $C^{\infty}$ topology. Since $\mathscr{S}_{n}$ includes all compactly supported $C^{\infty}$ functions, $\mathscr{S}_{n} \cap \mathscr{E}_{n}$ is weakly sequentially dense in $\mathscr{E}_{n}$. For $\mathfrak{G}_{\text {div }}$, one may do the following. When $s=n=3$, we have that $g=\nabla \times a$ for a $C^{\infty}$ vector field $a(x)$. Pick a $C^{\infty}$ cutoff function $\chi_{R}(x)$ that is 0 outside of a ball centered at the origin and having radius $R$, and 1 inside a ball centered at the origin and having radius $R / 2$. It is clear that the sequence $g_{k}(x)=\nabla \times\left\{\chi_{k}(x) a(x)\right\}$ is a compactly supported sequence that converges to $g$ not only weakly, but in the $C^{\infty}$ sense. For other $s$ or for $p$-forms, one may use an argument employing the converse of the Poincare lemma [7, p. 27].

As an example of our theory, let us construct an order-0 $\mathfrak{G}-\mathrm{CPD}$ matrixvalued function in the case where $\mathfrak{G}_{B}=\mathfrak{G}_{\text {div }}$. By the previous remark, the conditions in Theorem 6.1 on the admissible space $\mathfrak{G}_{\text {div }}$ are met. For this space, we have $s=n=3, \nu=1$, and $B_{1}(x)=B(x)=x$. Obviously, $B(-i \xi)=-i \xi$, and the projection onto $\{B(-i \xi)\}^{\perp}=\{\xi\}^{\perp}$ is given by $\Pi(\xi)=I-\|\xi\|^{-2} \xi \xi^{T}$ for all $\xi \neq 0$. We will simply take the polynomial part in (3.2) to be 0 . For $d \mu(\xi)$, we may take

$$
d \mu(\xi)=\left\{\|\xi\|^{2} I-\xi \xi^{T}\right\} e^{-\|\xi\|^{2} / 4 t} \frac{d^{3} \xi}{(4 \pi t)^{3 / 2}},
$$

where $t$ is some positive real number and $I$ is the identity matrix. It is straightforward to show that

$$
d \mu(\xi) \geq\|\xi\|^{2} \Pi(\xi) e^{-\|\xi\|^{2} / 4 t} \frac{d^{3} \xi}{(4 \pi t)^{3 / 2}},
$$


and so $d \mu$ satisfies (6.1). By Theorem 6.1, the function

$$
h_{t}^{3 \times 3}(x)=\int_{\mathbb{R}^{3}} e^{i x \cdot \xi}\left\{\|\xi\|^{2} I-\xi \xi^{T}\right\} e^{-\|\xi\|^{2} / 4 t} \frac{d^{3} \xi}{(4 \pi t)^{3 / 2}}
$$

is a strictly $\mathfrak{G}-\mathrm{CPD}$ of order 0 . The integral in (6.11) can be done, yielding the following explicit formula for the "divergence-free" CPDF $h_{t}^{3 \times 3}$ :

$$
h_{t}^{3 \times 3}(x)=\left\{\left(4 t-4 t^{2}\|x\|^{2}\right) I+4 t^{2} x x^{T}\right\} e^{-t\|x\|^{2}} .
$$

Of course, one can have $s$-dimensional divergence-free vector fields defined on $\mathbb{R}^{s}$. The $s \times s$ result corresponding to $(6.12)$ is

$$
h_{t}^{s \times s}(x)=\left\{\left(2(s-1) t-4 t^{2}\|x\|^{2}\right) I+4 t^{2} x x^{T}\right\} e^{-t\|x\|^{2}}
$$

When there are no side conditions-i.e., we are working in $\mathscr{E}_{n}$ itselfTheorem 6.1 provides us with the following result.

Corollary 6.3. Let $h$ be an order-m, matrix-valued CPDF having the form (3.2) . If the positive matrix-valued Borel measure $d \mu(\xi)$ in (3.2) satisfies $d \mu(\xi) \geq$ $I d \eta(\xi)$, where $I$ is the $n \times n$ identity matrix and $d \eta$ is a positive scalar-valued Borel measure that has support containing an open subset of $\mathbb{R}^{s}$, then $h$ is a strictly CPD matrix-valued function.

In the scalar case, the result above immediately reduces to the following one.

Corollary 6.4. Let $h$ be an order-m, scalar-valued CPDF having the form (3.2). If the positive scalar-valued Borel measure $d \mu(\xi)$ in (3.2) has support containing an open subset of $\mathbb{R}^{s}$, then $h$ is a strictly CPD scalar-valued function.

We remark that Corollary 6.4 and Theorem 2.3 imply that the class of Hermite interpolation problems treated in [26] is well poised. In the next section, we will use the results developed above to study stability questions in specific problems.

\section{Stability eStimates FOR MUlTiVARIATE HeRMite INTER POlation}

In this section, we obtain estimates on the norm of the inverse of the interpolation matrix that arises in a certain restricted class of Hermite interpolation problems. The main result is that these estimates are governed by the same parameters as in the case of ordinary interpolation via radial basis functions [19, 20]. Namely, the norm of the inverse depends on the particular RBF, the dimension, and the minimal separation of the Hermite data, but not on the number or distribution of points. While the result itself covers only a restricted class of the Hermite interpolation problems that we have shown to be well poised, the methods employed in obtaining the result can be tailored to apply to other more general situations.

The problem that we will address concerns norm estimates on the inverse of the interpolation matrix associated with interpolating values and certain multiples of directional derivatives for a scalar-valued function defined on $\mathbb{R}^{s}$. For this interpolation problem, we have $n=1$, with the distributions generating the data taken to be $\delta$-functions and directional deriatives of $\delta$-functions. Specifically, let $\left\{\zeta_{j}\right\}_{j=1}^{N}$ be $N$ distinct points in $\mathbb{R}^{s}$ with minimum separation $2 q$, and set

$$
\phi_{j}(x):=\delta\left(x-\zeta_{j}\right) \text { and } \psi_{j}:=v_{j} \cdot \nabla \delta\left(x-\zeta_{j}\right),
$$


for $j=1, \ldots, N$. Here, each $v_{j} \in \mathbb{R}^{s}$ is an arbitrary vector having a fixed length $L>0$; that is, $\left\|v_{j}\right\|=L$. We use the parameter " $L$ " for the common length of the $v_{j}$ 's, rather than using unit vectors, for two reasons. First, in a physical problem the "units" will be easier to deal with, and, second, the choice of $L$ can serve to precondition the interpolation matrix arising in the problem being addressed here.

What shall we take for our CPDF? We will not require polynomial reproduction, so we may take the order $m$ to be 0 . Recall that the radially symmetric, scaled Gaussian $h_{t}(x)=e^{-t\|x\|^{2}}$ has the representation

$$
h_{t}(x):=e^{-t\|x\|^{2}}=\int_{\mathbb{R}^{s}} e^{i x \cdot \xi} d \mu_{t}(\xi), \quad \text { where } d \mu_{t}(\xi):=e^{-\|\xi\|^{2} / 4 t} \frac{d^{s} \xi}{(4 \pi t)^{s / 2}},
$$

which is of the form (3.2) with measure $d \mu_{t}$ and $m=0$; it is thus an order-0 CPDF. The Gaussians are important because of Schoenberg's characterization of all order- 0 , radially symmetric CPDFs in terms of integrals (in $t$ ) of them [23, Theorem 6]. In view of that importance, we will use $h_{t}$ as our CPDF; that is, we will employ it in creating the $2 N \times 2 N$ interpolation matrix, $A_{t}$, for the problem described above. Our intent here is to demonstrate that $\left\|A_{t}^{-1}\right\|_{2} \leq \lambda(t, q, L, s)$ for some function $\lambda$ that is independent of $N$. Once the bound for the matrix $A_{t}^{-1}$ has been obtained, bounds for matrices associated with other CPDFs can be found using ideas employed in [20].

The starting point for obtaining bounds on the matrix $A_{t}^{-1}$ is the observation that the interpolation matrix $A_{t}$, which is defined in (2.5), is positive definite. This follows from Proposition 2.4 and the fact that $h_{t}$ is strictly CPD. Estimating $\left\|A_{t}^{-1}\right\|$ then amounts to getting a lower bound on the lowest eigenvalue of $A_{t}$, and then taking reciprocals.

To estimate the lowest eigenvalue of $A_{t}$, we need to look at the quadratic form $c^{*} A_{t} c$. Here, we write $c=\left(\begin{array}{llllll}a_{1} & \ldots & a_{N} & b_{1} & \ldots & b_{N}\end{array}\right)^{T}$. The first $N$ components correspond to $\phi_{j}$ 's, and the last $N$ to $\psi_{j}$ 's. From (2.5), (3.3), and (7.1), we have that

$$
c^{*} A_{t} c=\int_{\mathbb{R}^{s}}|\hat{\omega}(\xi)|^{2} d \mu_{t}(\xi), \quad \text { where } \omega=\sum_{j=1}^{N}\left(a_{j} \phi_{j}+b_{j} \psi_{j}\right) .
$$

We may put (7.2) into the form

$$
c^{*} A_{t} c=\|\hat{\omega}\|_{\mu_{t}}^{2},
$$

where $\|\cdot\|_{\mu_{t}}$ indicates the usual norm in $L^{2}\left(\mu_{t}\right)$. (A similar convention will be used for $L^{2}$ inner products.) If $d \nu_{t}(\xi)$ is any positive measure whose support contains an open subset of $\mathbb{R}^{s}$, and if $d \nu_{t}(\xi) \leq d \mu_{t}(\xi)$, then $c^{*} A_{t} c=\|\hat{\omega}\|_{\mu_{t}}^{2} \geq$ $\|\hat{\omega}\|_{\nu_{t}}^{2}$. Expanding $\|\hat{\omega}\|_{\nu_{t}}^{2}$ thus gives us

$$
c^{*} A_{t} c \geq \sum_{j, k=1}^{N}\left(\bar{a}_{j} a_{k}\left\langle\hat{\phi}_{k}, \hat{\phi}_{j}\right\rangle_{\nu_{t}}+\bar{b}_{j} b_{k}\left\langle\hat{\psi}_{k}, \hat{\psi}_{j}\right\rangle_{\nu_{t}}+\bar{a}_{j} b_{k}\left\langle\hat{\psi}_{k}, \hat{\phi}_{j}\right\rangle_{\nu_{t}}+\bar{b}_{j} a_{k}\left\langle\hat{\phi}_{k}, \hat{\psi}_{j}\right\rangle_{\nu_{t}}\right) .
$$

Breaking up part of the sum above into terms for which $j=k$ and terms for which $j \neq k$, and using the inequality $2|a b| \leq|a|^{2}+|b|^{2}$ in conjunction with 
the last inequality, we arrive at the following lower bound for $c^{*} A_{t} c$ :

$$
\begin{aligned}
c^{*} A_{t} c \geq & \sum_{j=1}^{N}\left|a_{j}\right|^{2}\left(\left\|\hat{\phi}_{j}\right\|_{\nu_{t}}^{2}-\sum_{k \neq j}\left|\left\langle\hat{\phi}_{k}, \hat{\phi}_{j}\right\rangle_{\nu_{t}}\right|-\sum_{k}\left|\left\langle\hat{\phi}_{j}, \hat{\psi}_{k}\right\rangle_{\nu_{t}}\right|\right) \\
& +\sum_{j=1}^{N}\left|b_{j}\right|^{2}\left(\left\|\hat{\psi}_{j}\right\|_{\nu_{t}}^{2}-\sum_{k \neq j}\left|\left\langle\hat{\psi}_{k}, \hat{\psi}_{j}\right\rangle_{\nu_{t}}\right|-\sum_{k}\left|\left\langle\hat{\phi}_{k}, \hat{\psi}_{j}\right\rangle_{\nu_{t}}\right|\right) .
\end{aligned}
$$

So far we have left $d \nu_{t}$ unspecified, except for the conditions imposed upon it earlier. Let us now choose $d \nu_{t}:=\hat{\chi}_{t}(\xi) d^{s} \xi$, where we assume that $0 \leq$ $\hat{\chi}_{t}(\xi) \leq e^{-\|\xi\|^{2} / 4 t} /(4 \pi t)^{s / 2}$ and that $\hat{\chi}_{t}$ is compactly supported, with its support containing some open set in $\mathbb{R}^{s}$. The inverse Fourier transform of $\hat{\chi}_{t}$ is $\chi_{t}(x)$. Since $\hat{\chi}_{t}$ is compactly supported, $\chi_{t}(x)$ will be an entire function of $x$, and we may freely differentiate it. Using $d \nu_{t}=\hat{\chi}_{t}(\xi) d^{s} \xi$ and the definitions of $\phi_{j}$ 's and $\psi_{j}$ 's, one can easily show that

$$
\begin{aligned}
& \left\langle\hat{\phi}_{k}, \hat{\phi}_{j}\right\rangle_{\nu_{t}}=\int_{\mathbf{R}^{s}} e^{i\left(\zeta_{j}-\zeta_{k}\right) \cdot \xi} \hat{\chi}_{t}(\xi) d^{s} \xi=(2 \pi)^{s} \chi_{t}\left(\zeta_{j}-\zeta_{k}\right), \\
& \left\langle\hat{\psi}_{k}, \hat{\psi}_{j}\right\rangle_{\nu_{t}}=\int_{\mathbf{R}^{s}} e^{i\left(\zeta_{j}-\zeta_{k}\right) \cdot \xi}\left(v_{j} \cdot \xi\right)\left(v_{k} \cdot \xi\right) \hat{\chi}_{t}(\xi) d^{s} \xi=-(2 \pi)^{s} v_{j}^{T} \chi_{t}^{\prime \prime}\left(\zeta_{j}-\zeta_{k}\right) v_{k}, \\
& \left\langle\hat{\phi}_{k}, \hat{\psi}_{j}\right\rangle_{\nu_{t}}=\int_{\mathbf{R}^{s}} e^{i\left(\zeta_{j}-\zeta_{k}\right) \cdot \xi} i\left(v_{j} \cdot \xi\right) \hat{\chi}_{t}(\xi) d^{s} \xi=(2 \pi)^{s} v_{j}^{T} \chi_{t}^{\prime}\left(\zeta_{j}-\zeta_{k}\right),
\end{aligned}
$$

where $\chi_{t}^{\prime}(x)$ denotes the column vector representing the gradient of $\chi_{t}$ and $\chi_{t}^{\prime \prime}(x)$ denotes a symmetric, $s \times s$ matrix representing the Hessian of $\chi_{t}$. If we use these expressions in (7.4), then we get

$$
\begin{aligned}
c^{*} A_{t} c \geq & \sum_{j=1}^{N}(2 \pi)^{s}\left|a_{j}\right|^{2}\left\{\chi_{t}(0)-\sum_{k \neq j}\left|\chi_{t}\left(\zeta_{j}-\zeta_{k}\right)\right|-\sum_{k}\left|v_{k}^{T} \chi_{t}^{\prime}\left(\zeta_{k}-\zeta_{j}\right)\right|\right\} \\
& +\sum_{j=1}^{N}(2 \pi)^{s}\left|b_{j}\right|^{2}\left\{\left|v_{j}^{T} \chi_{t}^{\prime \prime}(0) v_{j}\right|-\sum_{k \neq j}\left|v_{j}^{T} \chi_{t}^{\prime \prime}\left(\zeta_{j}-\zeta_{k}\right) v_{k}\right|\right. \\
& \left.-\sum_{k}\left|v_{j}^{T} \chi_{t}^{\prime}\left(\zeta_{j}-\zeta_{k}\right)\right|\right\} .
\end{aligned}
$$

Since $\left\|v_{j}\right\|=L$, we have

$$
\left|v_{k}^{T} \chi_{t}^{\prime}\left(\zeta_{k}-\zeta_{j}\right)\right| \leq L\left\|\chi_{t}^{\prime}\left(\zeta_{j}-\zeta_{k}\right)\right\|
$$

and

$$
\left|v_{j}^{T} \chi_{t}^{\prime \prime}\left(\zeta_{j}-\zeta_{k}\right) v_{k}\right| \leq L^{2}\left\|\chi_{t}^{\prime \prime}\left(\zeta_{j}-\zeta_{k}\right)\right\|,
$$

where $\|\cdot\|$ denotes the appropriate vector or matrix norm in $l^{2}$. Inserting these 
norm inequalities in the preceding inequality yields

$$
\begin{aligned}
c^{*} A_{t} c \geq & \sum_{j=1}^{N}(2 \pi)^{s}\left|a_{j}\right|^{2}\left\{\chi_{t}(0)-\sum_{k \neq j}\left|\chi_{t}\left(\zeta_{j}-\zeta_{k}\right)\right|-\sum_{k} L\left\|\chi_{t}^{\prime}\left(\zeta_{k}-\zeta_{j}\right)\right\|\right\} \\
+ & \sum_{j=1}^{N}(2 \pi)^{s}\left|b_{j}\right|^{2}\left\{\left|v_{j}^{T} \chi_{t}^{\prime \prime}(0) v_{j}\right|-\sum_{k \neq j} L^{2}\left\|\chi_{t}^{\prime \prime}\left(\zeta_{j}-\zeta_{k}\right)\right\|\right. \\
& \left.-\sum_{k} L\left\|\chi_{t}^{\prime}\left(\zeta_{j}-\zeta_{k}\right)\right\|\right\} .
\end{aligned}
$$

The choice of $\hat{\chi}_{t}$ (and, of course, $\chi_{t}$ as well) is still restricted only by the requirements that

$$
0 \leq \hat{\chi}_{t}(\xi) \leq \frac{e^{-\|\xi\|^{2} / 4 t}}{(4 \pi t)^{s / 2}}
$$

and that $\hat{\chi}_{t}$ be compactly supported with its support containing an open subset of $\mathbb{R}^{s}$. We will now exhibit a one-parameter family of such functions.

Recall the definition of the $p$ th-order $B$-spline (of a single variable) $\mathfrak{B}_{p}(u)$. If $\mathfrak{B}_{1}(u)$ denotes the characteristic function of $[-1 / 2,1 / 2]$, then

$$
\mathfrak{B}_{p}:=\underbrace{\mathfrak{B}_{1} * \cdots * \mathfrak{B}_{1}}_{p \text {-fold }} .
$$

The tensor product spline is defined as $T_{p}(\xi):=\prod_{k=1}^{s} \mathfrak{B}_{p}\left(\xi_{k}\right)$, where $\xi_{k}$ is the $k$ th component of $\xi$. From standard properties of $\mathfrak{B}_{p}$ (see [3]), we see that $T_{p}$ has these properties:

(a) $T_{p}$ is an even, piecewise polynomial function;

(b) $T_{p}(\xi)>0$ for every $\xi$ in the $s$-cube $-p / 2<\xi_{k}<p / 2$ and is zero elsewhere;

(c) $T_{p}(\xi) \leq T_{p}(0)=\mathfrak{B}_{p}(0)^{s}$;

(d) $\widehat{T}_{p}=\prod_{k=1}^{s} \operatorname{sinc}^{p}\left(x_{k}\right)$;

(e) $T_{p}=\left\{(2 \pi)^{-s} \prod_{k=1}^{s} \operatorname{sinc}^{p}\left(x_{k}\right)\right\}^{\wedge}$.

The equation in (e) above is the consequence of (d) and a Fourier transform identity.

Lemma 7.1. Let $\rho$ be any positive number, and set

$$
c_{t, \rho}:=\frac{e^{-s p^{2} /\left(16 \rho^{2} t\right)}}{\left(16 \pi^{3} t \rho^{2} \mathfrak{B}_{p}(0)^{2}\right)^{s / 2}} .
$$

Each member of the family of functions defined by

$$
\hat{\chi}_{t, \rho}(\xi):=(2 \pi \rho)^{s} c_{t, \rho} T_{p}(\rho \xi)
$$

satisfies (7.6), is positive on the s-cube $-p /(2 \rho)<\xi_{k}<p /(2 \rho)$, and is zero outside of this s-cube. The inverse Fourier transform of $\hat{\chi}_{t, \rho}$ is given by

$$
\chi_{t, \rho}(x)=c_{t, \rho} \prod_{k=1}^{s} \operatorname{sinc}^{p}\left(x_{k} / \rho\right),
$$

where $x_{k}$ denotes the kth component of $x$. 
Proof. The result follows from combining the various properties of $T_{p}$ listed above with the observation that the minimum of $e^{-\|\xi\|^{2} / 4 t}$ on the $s$-cube described above occurs at $\xi_{k}= \pm p /(2 \rho)$.

We need to estimate the quantities found in (7.5), given that (7.8) is our choice for $\chi_{t}$. From (7.8), we can obtain all first and second partials of $\chi_{t, \rho}$. The first-order partials are

$$
\frac{\partial \chi_{t, \rho}}{\partial x_{j}}(x)=\frac{p c_{t, \rho}}{\rho} \operatorname{sinc}^{p-1}\left(x_{j} / \rho\right) \operatorname{sinc}^{\prime}\left(x_{j} / \rho\right) \prod_{k \neq j} \operatorname{sinc}^{p}\left(x_{k} / \rho\right) .
$$

We can differentiate (7.9) to obtain

$$
\begin{aligned}
\frac{\partial^{2} \chi_{t, \rho}}{\partial x_{j} \partial x_{i}}(x)= & \frac{p^{2} c_{t, \rho}}{\rho^{2}} \operatorname{sinc}^{p-1}\left(x_{j} / \rho\right) \operatorname{sinc}^{p-1}\left(x_{i} / \rho\right) \operatorname{sinc}^{\prime}\left(x_{j} / \rho\right) \\
& \cdot \operatorname{sinc}^{\prime}\left(x_{i} / \rho\right) \prod_{k \neq i, j} \operatorname{sinc}^{p}\left(x_{k} / \rho\right) \quad \text { if } i \neq j .
\end{aligned}
$$

When $i=j$, we have this expression:

$$
\begin{aligned}
\frac{\partial^{2} \chi_{t, \rho}}{\partial x_{j}^{2}}(x)=\frac{p c_{t, \rho}}{\rho^{2}}\{(p-1) & \operatorname{sinc}^{p-2}\left(x_{j} / \rho\right)\left[\operatorname{sinc}^{\prime}\left(x_{j} / \rho\right)\right]^{2} \\
& \left.+\operatorname{sinc}^{p-1}\left(x_{j} / \rho\right) \operatorname{sinc}^{\prime \prime}\left(x_{j} / \rho\right)\right\} \prod_{k \neq j} \operatorname{sinc}^{p}\left(x_{k} / \rho\right) .
\end{aligned}
$$

From (7.8)-(7.11) and the properties of $\operatorname{sinc}(\cdot)$, we can easily compute various derivatives of $\chi_{t, \rho}$ at $x=0$; the results of these computations are the following formulas:

$$
\begin{gathered}
\chi_{t, \rho}(0)=c_{t, \rho} \quad \text { and } \quad \frac{\partial \chi_{t, \rho}}{\partial x_{j}}(0)=0 \\
\frac{\partial^{2} \chi_{t, \rho}}{\partial x_{j}^{2}}(0)=-\frac{p c_{t, \rho}}{12 \rho^{2}} \quad \text { and } \quad \frac{\partial^{2} \chi_{t, \rho}}{\partial x_{j} \partial x_{i}}(0)=0 \quad \text { if } i \neq j .
\end{gathered}
$$

The function $\operatorname{sinc}(\cdot)$ plays a crucial role in our computations, and we need to estimate it and its derivatives up to second order. This we do in the next result.

Lemma 7.2. There holds

$$
\left|\operatorname{sinc}^{(l)}(v)\right| \leq \begin{cases}\min \left\{1, \frac{2}{|v|}\right\} & \text { if } l=0, \\ \min \left\{\frac{1}{l+1}\left(\frac{1}{2}\right)^{l},\left(\frac{1}{2}\right)^{l-2} \frac{1}{|v|}\right\} \leq \min \left\{1, \frac{2}{|v|}\right\} & \text { if } l \geq 1 .\end{cases}
$$

Proof. Using the Fourier integral representation for $\operatorname{sinc}(\cdot)$, we have

$$
\left|\operatorname{sinc}^{(l)}(v)\right| \leq\left|\int_{-1 / 2}^{1 / 2} u^{l} e^{i u v} d u\right| \leq \int_{-1 / 2}^{1 / 2}|u|^{l} d u=\frac{1}{l+1}\left(\frac{1}{2}\right)^{l} .
$$

Applying Leibniz's rule for the $l$ th derivative of a product $l$ times to $v \operatorname{sinc}(v)$ $=2 \sin (v / 2)$ yields

$$
v \operatorname{sinc}^{(l)}(v)+l \operatorname{sinc}^{(l-1)}(v)= \pm\left(\frac{1}{2}\right)^{l-1}\left\{\begin{array}{l}
\sin (v / 2), \\
\cos (v / 2) .
\end{array}\right.
$$


After rearranging terms, dividing by $v$, and taking absolute values in the last equation, we easily see that

$$
\left|\operatorname{sinc}^{(l)}(v)\right| \leq \frac{1}{|v|}\left(\frac{1}{2}\right)^{l-1}+\frac{l\left|\operatorname{sinc} c^{(l-1)}(v)\right|}{|v|}
$$

Using (7.15) with $l \rightarrow l-1$ to replace the numerator of the term on the far right, we get

$$
\left|\operatorname{sinc}^{(l)}(v)\right| \leq|v|^{-1} 2^{2-l} .
$$

Combining (7.15) with the inequality above yields (7.14).

A direct application of (7.14) to (7.8)-(7.11) yields these estimates for $\chi_{t, \rho}$ and its first and second partials:

$$
\left|\partial^{\alpha} \chi_{t, \rho}(x)\right| \leq c_{t, \rho}\left(\frac{p}{\rho}\right)^{|\alpha|} \prod_{k=1}^{s}\left[\min \left\{1, \frac{2 \rho}{\left|x_{k}\right|}\right\}\right]^{p}, \quad \text { where } 0 \leq|\alpha| \leq 2
$$

In (7.16), suppose that $x \neq 0$ and that $\max _{k}\left\{\left|x_{k}\right|\right\}=\left|x_{k_{0}}\right|$. A standard argument then implies that $\left|x_{k_{0}}\right| \geq\|x\| / \sqrt{s}$. If in (7.16) we use $\min \left\{1,2 \rho /\left|x_{k}\right|\right\} \leq 1$ when $k \neq k_{0}$ and we use $\min \left\{1,2 \rho /\left|x_{k_{0}}\right|\right\} \leq 2 \rho /\left|x_{k_{0}}\right| \leq 2 \rho \sqrt{s} /\|x\|$, then (7.16) becomes

$$
\left|\partial^{\alpha} \chi_{t, \rho}(x)\right| \leq c_{t, \rho}\left(\frac{p}{\rho}\right)^{|\alpha|}\left[\frac{2 \rho \sqrt{s}}{\|x\|}\right]^{p}, \quad \text { where } 0 \leq|\alpha| \leq 2 \text { and } x \neq 0 .
$$

The derivative estimates in (7.17) can be used in combination with standard vector- and matrix-norm estimates to bound the gradient and Hessian of $\chi_{t, \rho}$. We state the estimates obtained in this way below.

Lemma 7.3. If $x \neq 0$, then

$$
\left\{\begin{array}{l}
\left|\chi_{t, \rho}(x)\right| \leq c_{t, \rho}\left[\frac{2 \rho \sqrt{s}}{\|x\|}\right]^{p}, \\
\left|\chi_{t, \rho}^{\prime}(x)\right| \leq \sqrt{s} c_{t, \rho}\left(\frac{p}{\rho}\right)\left[\frac{2 \rho \sqrt{s}}{\|x\|}\right]^{p}, \\
\left|\chi_{t, \rho}^{\prime \prime}(x)\right| \leq s c_{t, \rho}\left(\frac{p}{\rho}\right)^{2}\left[\frac{2 \rho \sqrt{s}}{\|x\|}\right]^{p} .
\end{array}\right.
$$

The estimates in (7.18) and the values for $\chi_{t, \rho}$ and its derivatives in (7.12) and (7.13) may be used together with the lower bound (7.5) for $c^{*} A_{t} c$ to obtain

$$
\frac{c^{*} A_{t} c}{(2 \pi)^{s} c_{t, \rho}} \geq \sum_{j=1}^{N}\left|a_{j}\right|^{2}\left\{1-(2 \rho \sqrt{s})^{p}\left[1+p \sqrt{s} L \rho^{-1}\right] \sum_{k \neq j} \frac{1}{\left\|\zeta_{j}-\zeta_{k}\right\|^{p}}\right\}
$$

$$
+\sum_{j=1}^{N}\left|b_{j}\right|^{2}\left\{\frac{p L^{2}}{12 \rho^{2}}-p \sqrt{s} L \rho^{-1}(2 \rho \sqrt{s})^{p}\left[1+p \sqrt{s} L \rho^{-1}\right]\right.
$$

$$
\left.\sum_{k \neq j} \frac{1}{\left\|\zeta_{j}-\zeta_{k}\right\|^{p}}\right\}
$$

To estimate $\sum_{k \neq j}\left(1 /\left\|\zeta_{j}-\zeta_{k}\right\|^{p}\right)$, repeat the "packing" argument employed in $[19$, p. 80$]$ to get

$$
\sum_{k \neq j} \frac{1}{\left\|\zeta_{j}-\zeta_{k}\right\|^{p}} \leq 3^{s} \sum_{n=1}^{\infty} n^{s-1} \kappa_{n}
$$


where $\kappa_{n}:=\sup \left\{\|x\|^{-p}: n q \leq\|x\| \leq(n+1) q\right\}$ and $2 q$ is the minimal separation distance for $\left\{\zeta_{j}\right\}_{j=1}^{N} \subset \mathbb{R}^{s}$. Clearly, we have that $\kappa_{n}=(n q)^{-p}$. Using this above yields

$$
\sum_{k \neq j} \frac{1}{\left\|\zeta_{j}-\zeta_{k}\right\|^{p}} \leq 3^{s} q^{-p} \sum_{n=1}^{\infty} n^{s-1-p} .
$$

If we take $p \geq s+1$, we see that the series on the right is bounded above by $\sum_{n \geq 1} n^{-2}=\pi^{2} / 6$, and so

$$
\sum_{k \neq j} \frac{1}{\left\|\zeta_{j}-\zeta_{k}\right\|^{p}} \leq\left(3^{s} q^{-p}\right) \frac{\pi^{2}}{6}<3^{s+1} q^{-p} .
$$

Using the inequality above in (7.19), taking $p \geq s+1$, and factoring the coefficient of the $b_{j}$ 's, we arrive at the following inequality:

$$
\begin{aligned}
\frac{c^{*} A_{t} c}{(2 \pi)^{s} c_{t, \rho}} \geq & \sum_{j=1}^{N}\left|a_{j}\right|^{2}\left\{1-3^{s+1}\left(2 \rho q^{-1} \sqrt{s}\right)^{p}\left[1+p \sqrt{s} L \rho^{-1}\right]\right\} \\
& +\sum_{j=1}^{N}\left|b_{j}\right|^{2} \frac{p L^{2}}{12 \rho^{2}}\left\{1-\left(12 \sqrt{s} \rho L^{-1}\right) 3^{s+1}\left(2 \rho q^{-1} \sqrt{s}\right)^{p}\left[1+p \sqrt{s} L \rho^{-1}\right]\right\}
\end{aligned}
$$

It is clear that we are able to choose $\rho>0$ so that

$$
12 \sqrt{s} \rho L^{-1} \leq 1 \text { and } 3^{s+1}\left(2 \rho q^{-1} \sqrt{s}\right)^{p}\left[1+p \sqrt{s} L \rho^{-1}\right] \leq 1 / 2 .
$$

If we do so, then, from (7.20), the assumption that $p \geq s+1$, and the fact that $s \geq 1$, we easily see that

$$
\frac{c^{*} A_{t} c}{(2 \pi)^{s} c_{t, \rho}} \geq \frac{1}{2}\|c\|^{2}
$$

from which the theorem below follows immediately.

Theorem 7.4. Let $p \geq s+1$, choose $\rho=\rho(q, p, s, L)>0$ to satisfy the inequalities in (7.21), and let $d \beta(t)$ be a finite, nonnegative Borel measure defined on $[0, \infty)$ with support including some nonempty subset of $(0, \infty)$. If $h(x)$ and $A$ have the form

$$
h(x):=\int_{0}^{\infty} e^{-t\|x\|^{2}} d \beta(t) \text { and } A:=\int_{0}^{\infty} A_{t} d \beta(t),
$$

then

(7.23) $\quad c^{*} A c \geq \theta(q, s, p, L)\|c\|^{2} \quad$ where $\theta:=\frac{1}{2} \int_{0}^{\infty} \frac{e^{-s p^{2} /\left(16 \rho^{2} t\right)}}{\left(4 \pi t \rho^{2} \mathfrak{B}_{p}(0)^{2}\right)^{s / 2}} d \beta(t)$.

Proof. The lower bound (7.23) follows from first multiplying both sides of (7.22) by $c_{t, \rho}$, then replacing $c_{t, \rho}$ in (7.22) with the expression found in (7.7), and finally integrating the result with respect to $d \beta$.

The matrix $A$ is of course the interpolation matrix for the problem described at the beginning of this section, provided the CPDF used is the $h$ defined in Theorem 7.4. We can now precisely state the estimate for $\left\|A^{-1}\right\|$ that we promised earlier. 
Corollary 7.5. With the notation and assumptions of Theorem 7.4, we have that

$$
\left\|A^{-1}\right\| \leq \theta^{-1} \text {. }
$$

Proof. As a consequence of (7.23), the lowest eigenvalue of $A$ is bounded below by $\theta$. Using the fact that $\left\|A^{-1}\right\|$ is the reciprocal of the lowest eigenvalue yields (7.24).

The bound (7.24) does not depend on $N$, which is the number of data sites. As promised, it does depend on the length scales involved, $q$ and $L$, and also on both $p$, the order of the $B$-spline used, and $s$, the dimension of the ambient space. (By choosing $p=s+1$, one may remove the dependence on $p$.) This behavior is similar to what was found in [20] for the problem in which only function values are interpolated. For certain order-one CPDFs (see [20]), one may also use (7.22) to obtain formulas similar to those in Theorem 7.4 and Corollary 7.5. Indeed, one can use (7.22) with $b_{j}=0, j=1, \ldots, N$, to recover an estimate similar to that given in [20, Theorem $\left.2.3^{1}\right]$; only the constants used are different.

We remark that the Hermite interpolation problem for which we have derived our estimates required as data both value and derivative along a vector at each data site. The line of reasoning used, however, can easily be applied to the case in which one has as data either value or derivative along a vector or both at each data site. The resulting estimates are unchanged.

\section{ACKNOWLEDGMENT}

We wish to thank Brad Baxter and the referee for helpful suggestions.

\section{BIBLIOGRAPHY}

1. R. Beale and T. Jackson, Neural computing: An introduction, Adam Hilger, Bristol, UK, 1990.

2. S. K. Berberian, Notes on spectral theory, Van Nostrand, Princeton, NJ, 1966.

3. C. de Boor, A practical guide to splines, Springer-Verlag, New York and Berlin, 1978.

4. M. D. Buhmann, New developments in the theory of radial basis function interpolation, Multivariate Approximation and Wavelets (K. Jetter and F. I. Uteras, eds.), World Scientific, Singapore, 1992, pp. 1-39.

5. J. Duchon, Interpolation des fonctions de deux variables suivant le principe de la flexion des plaques minces, RAIRO Anal. Numér. 10 (1976), 5-12.

6. __ Splines minimizing rotation invariant semi-norms in Sobolev spaces, Constructive Theory of Functions of Several Variables, Oberwolfach 1976 (W. Schempp and K. Zeller, eds.), Springer-Verlag, Berlin, 1977, pp. 85-100.

7. H. Flanders, Differential forms, Academic Press, New York, 1963.

8. F. G. Friedlander, Introduction to the theory of distributions, Cambridge Univ. Press, Cambridge, 1982.

9. I. M. Gelfand and N. Ya. Vilenkin, Generalized functions, Vol. 4, Academic Press, New York, 1964.

10. R. L. Hardy, Multiquadric equations of topography and other irregular surfaces, J. Geophys. Res. 76 (1971), 1905-1915.

11. Appl. 19 (1990), 163-208.

${ }^{1}$ There is a typographical error in Theorem 2.3 in [20]: the constant $C_{s}$ is incorrectly given there. The correct form is found in [20, equation (3.22)]. 
12. K. Jetter, S. D. Riemenschneider, and Z. Shen, Hermite interpolation on the lattice $\mathbb{Z}^{d}$, SIAM J. Math. Anal. 25 (1994), 962-975.

13. E. J. Kansa, Multiquadrics-a scattered data approximation scheme with applications to computational fluid dynamics. I. Surface approximations and partial derivative estimates, Comput. Math. Appl. 19 (1990), 127-145.

14. __ Multiquadrics - a scattered data approximation scheme with applications to computational fluid dynamics. II. Solutions to parabolic, hyperbolic and elliptic partial differential equations, Comput. Math. Appl. 19 (1990), 147-161.

15. W. R. Madych and S. A. Nelson, Multivariate interpolation and conditionally positive definite functions, Approx. Theory Appl. 4 (1988), 77-79.

16. _ Multivariate interpolation aid conditionally positive definite functions. II, Math. Comp. 54 (1990), 211-230.

17. R. E. Meyer, Introduction to mathematical fluid dynamics, Dover, New York, 1982.

18. C. A. Micchelli, Interpolation of scattered data: distances, matrices, and conditionally positive definite functions, Constr. Approx. 2 (1986), 11-22.

19. F. J. Narcowich and J. D. Ward, Norms of inverses and condition numbers for matrices associated with scattered data, J. Approx. Theory 64 (1991), 69-94.

20. __ Norm estimates for the inverses of a general class of scattered-data radial-function interpolation matrices, J. Approx. Theory 69 (1992), 84-109.

21. T. O'Donnell, J. Simmers, and D. J. Jacavanco, Neural beamforming for phased array antennas, Proc. 1992 Antenna Applications Symposium (Robert Allerton Park, ed.), USAF HG Rome Laboratory, Griffis AFB, NY, 1992 (to appear).

22. T. Poggio and F. Girosi, $A$ theory of networks for approximating and learning, MIT Artificial Intelligence Laboratory and Center for Biological Information Processing (Whitaker College), A. I. Memo No. 1140, C.B.I.P. Paper No. 31, 1989.

23. I. Schoenberg, Metric spaces and completely monotone functions, Ann. of Math. (2) 39 (1938), 811-841.

24. R. M. Sanner and J.-J. E. Slotine, Gaussian networks for direct adaptive control, Proc. American Control Conference (Boston, MA, June, 1991), Vol. 3, The American Automatic Control Council, Evanston, IL, 1991, pp. 2153-2159.

25. F. Treves, Topological vector spaces, distributions, and kernels, Academic Press, New York, 1967.

26. Wu Zhongmin, Hermite-Birkhoff interpolation of scattered data by radial basis functions, Approx. Theory Appl. 8 (1992), 1-10.

Department of Mathematics, Texas A \& M University, College Station, Texas 778433368

E-mail address, F.-J. Narcowich: fnarc@math.tamu.edu

E-mail address, J. D. Ward: jward@math.tamu.edu 